\title{
Lower-dimensional nonlinear Brinkman's law for non-Newtonian flows in a thin porous medium
}

\author{
María ANGUIANO ${ }^{1}$, Francisco J. SUÁREZ-GRAU²
}

\begin{abstract}
In this paper we study the stationary incompressible power law fluid flow in a thin porous medium. The media under consideration is a bounded perforated 3D domain confined between two parallel plates, where the distance between the plates is very small. The perforation consists in an array solid cylinders, which connect the plates in perpendicular direction, distributed periodically with diameters of small size compared to the period. For a specific choice of the thickness of the domain, we found that the homogenization of the power law Stokes system results a lower-dimensional nonlinear Brinkman type law.
\end{abstract}

AMS classification numbers: $76 \mathrm{~A} 05,35 \mathrm{~B} 27,76 \mathrm{M} 50$.

Keywords: Homogenization, non-Newtonian fluid, power law fluid, thin porous medium, Brinkman's law.

\section{Introduction}

Modeling non-Newtonian fluid flows in porous medium is very important due to its practical engineering applications, such as oil recovery, food processing, and materials processing. Non-Newtonian fluids exhibit a nonlinear behavior that is different from that of Newtonian fluids and the related equations are too complicated to be solved in the porous medium, due to its complex geometry. Thus, macroscopic laws obtained by homogenization techniques are used as a good substitute for the original physical laws.

A relevant case of non-Newtonian fluid is the power law or Ostwald-de Waele model, which assumes that the viscosity satisfies a nonlinear power law. More precisely, if $u$ is the velocity field and $D u$ the gradient velocity tensor, denoting the shear rate by $\mathbb{D}[u]=\frac{1}{2}\left(D u+D^{t} u\right)$, the viscosity as a function of the shear rate is given by

$$
\eta_{r}(\mathbb{D}[u])=\mu|\mathbb{D}[u]|^{r-2}, \quad 1<r<+\infty .
$$

The two parameters $\mu>0$ and $r$ are called the consistency and the flow index, respectively. The matrix norm $|\cdot|$ is defined by $|\xi|^{2}=\operatorname{Tr}\left(\xi \xi^{t}\right)$ with $\xi \in \mathbb{R}^{3}$, so the viscosity is expressed in term of the second invariant of the strain tensor $\mathbb{D}_{I I}[u]=\mathbb{D}[u] \mathbb{D}[u]^{t}$ by

$$
|\mathbb{D}[u]|^{r-2}=\left|\mathbb{D}_{I I}[u]\right|^{\frac{r}{2}-1}
$$

We recall that $r=2$ yields the Newtonian fluid, for $r \in(1,2)$ the fluid is a shear-thinning and for $r \in(2,+\infty)$ is a shear-thickening.

The derivation of macroscopic law for power law fluids through periodic porous medium with a periodic arrangement of obstacles has been consider in Bourgeat et al. [8, 9]. Denoting $\varepsilon$ a small parameter related to the characteristic size of the obstacles and the period of the periodic structure and starting from the $3 \mathrm{D}$ power

\footnotetext{
${ }^{1}$ Departamento de Análisis Matemático. Facultad de Matemáticas. Universidad de Sevilla, 41012 Sevilla (Spain) anguiano@us.es

${ }^{2}$ Departamento de Ecuaciones Diferenciales y Análisis Numérico. Facultad de Matemáticas. Universidad de Sevilla, 41012 Sevilla (Spain) grau@us.es
} 
law Stokes system with body forces $f$, by means of the two-scale convergence method when $\varepsilon$ tends to zero, the following nonlinear Darcy type law for the pressure $p$ is derived in an $\varepsilon$-independent domain $\Omega=\omega \times(0,1) \subset \mathbb{R}^{3}$

$$
\left\{\begin{array}{l}
u=\frac{1}{\mu} \mathcal{U}(f-\nabla p) \quad \text { in } \Omega, \\
\operatorname{div} u=0 \quad \text { in } \Omega, \quad u \cdot n=0 \quad \text { on } \partial \Omega .
\end{array}\right.
$$

The nonlinear function $\mathcal{U}: \mathbb{R}^{3} \rightarrow \mathbb{R}^{3}$ is called permeability function of the porous medium and is defined through the solutions of auxiliary problems of power law type depending on the geometrical structure of the domain. We refer to Mikelić $[20,21]$ for a complete review on the modeling of non-Newtonian flows in porous medium by using homogenization.

A more general case of periodic porous medium is when the size of the solid obstacles is assumed to be much smaller than the period. Thus, denoting $\varepsilon$ the period of the periodic porous structure and considering obstacles of size $\varepsilon \delta$ with $\delta_{\varepsilon}$ a parameter which depends on the period and changes simultaneously during the homogenization process, it is well-known that there exists a critical regime in which the macroscopic law describing the behavior of Newtonian fluids is the Brinkman's law instead of the Darcy's law, see Allaire [1,2] and Brillard [10]. This change of behavior occurs for power law fluids as well. More precisely, assuming that the relation $\sigma_{\varepsilon}=\varepsilon / \delta_{\varepsilon}^{\frac{3-r}{r}}$ tends to a positive constant when $\varepsilon$ tends to zero, Fratrović and Marušić-Paloka [15] derived by means of $\Gamma$-convergence the following nonlinear Brinkman type law

$$
\left\{\begin{array}{l}
-\mu \operatorname{div}\left(|\mathbb{D}[u]|^{r-2} \mathbb{D}[u]\right)+\mu \mathcal{G}(u)+\nabla p=f \text { in } \Omega \\
\operatorname{div} u=0 \text { in } \Omega \\
u=0 \quad \text { on } \partial \Omega
\end{array}\right.
$$

where the nonlinear function $\mathcal{G}: \mathbb{R}^{3} \rightarrow \mathbb{R}^{3}$ is called the drag force function of the porous medium and it provides the value of the drag force on the reference obstacle. Moreover, it is defined through the solution of auxiliary exterior problems of power law type depending on the geometrical structure of the obstacles. We remark that due to technical reasons, such as the derivation of a sharp Poincaré-Korn inequality and the existence of a unique solution of exterior auxiliary problems in homogeneous Sobolev spaces, the fluid index $r$ has to be restricted, i.e. be smaller than the dimension of the domain. In fact, this result is only proved to shear thinning fluids.

On the other hand, the derivation of macroscopic laws for fluid flows in a thin porous medium has recently become of great interest, see Anguiano and Suárez-Grau [5] and Fabricius et al. [13]. More precisely, a thin porous medium can be defined as a bounded perforated 3D domain confined between two parallel plates, where the distance between the plates is very small and the perforation consists of periodically distributed solid cylinders which connect the plates in perpendicular direction. In previous studies, the thin porous medium is described by two parameters: one parameter $\varepsilon$ denoting the period of the periodic porous structure as well as the size of the diameter of the cylinders, and another parameter $h_{\varepsilon}$ denoting the thickness of the domain. Thus, the derivation of macroscopic laws through a thin porous medium depends on the relation between both parameters. Concerning power law fluids, in a recent article (see [4]) we derived different lower-dimensional forms of the nonlinear Darcy's law in $\omega \subset \mathbb{R}^{2}$ by using an adaptation of the unfolding method depending on the parameter $\varepsilon$, which can be written as follows

$$
\left\{\begin{array}{l}
u^{\prime}=\frac{1}{\mu} \mathcal{U}\left(f^{\prime}-\nabla_{x^{\prime}} p\right), \quad u_{3}=0 \quad \text { in } \omega \\
\operatorname{div}_{x^{\prime}} u^{\prime}=0 \quad \text { in } \omega, \quad u^{\prime} \cdot n=0 \quad \text { on } \partial \omega .
\end{array}\right.
$$

Here $u^{\prime}=\left(u_{1}, u_{2}\right), f^{\prime}=\left(f_{1}, f_{2}\right), x^{\prime}=\left(x_{1}, x_{2}\right)$ and the permeability function $\mathcal{U}: \mathbb{R}^{2} \rightarrow \mathbb{R}^{2}$ is defined through the solutions of lower-dimensional auxiliary problems of power law type.

The goal of this paper is to study the homogenization of power law fluids in thin porous medium containing an array of periodically perforated cylinders with diameters much smaller than the period. As far as we know, 
this problem has never been considered in the previous literature. This domain is then defined by three small parameters denoted by $\varepsilon, \delta_{\varepsilon}$ and $h_{\varepsilon}$, where $\varepsilon$ denotes the period of the periodic porous structure, $\varepsilon \delta_{\varepsilon}$ the size of the diameter of the cylinders and $h_{\varepsilon}$ the thickness of the domain. For an appropriate choice (critical size) of $h_{\varepsilon}$, we derive in $\Omega$ the following lower-dimensional nonlinear Brinkman type law

$$
\left\{\begin{array}{l}
-\mu 2^{-\frac{r}{2}} \partial_{x_{3}}\left(\left|\partial_{x_{3}} u^{\prime}\right|^{r-2} \partial_{x_{3}} u^{\prime}\right)+\mu \mathcal{G}\left(u^{\prime}\right)+\nabla_{x^{\prime}} p=f^{\prime}, \quad u_{3}=0 \quad \text { in } \Omega, \\
\operatorname{div}_{x^{\prime}}\left(\int_{0}^{1} u^{\prime} d x_{3}\right)=0 \quad \text { in } \omega, \quad\left(\int_{0}^{1} u^{\prime} d x_{3}\right) \cdot n=0 \quad \text { on } \partial \omega, \\
u^{\prime}\left(x^{\prime}, 0\right)=u^{\prime}\left(x^{\prime}, 1\right)=0 \text { in } \omega,
\end{array}\right.
$$

where the nonlinear drag force function $\mathcal{G}: \mathbb{R}^{2} \rightarrow \mathbb{R}^{2}$ is defined through the solution of lower-dimensional auxiliary exterior problems of power law type depending on the geometrical structure of the obstacles. The result is obtained by using a combination of reduction of dimension techniques, monotonicity arguments and an adaptation of the unfolding method depending on parameters $\varepsilon$ and $\delta_{\varepsilon}$. We also remark that due to different technical reasons, which will be noted along the paper, we have to restrict this study to shear thinning power law fluids. For instance, in order to obtain optimal a priori estimates of the solution, we need to use Poincaré-Korn's and a Sobolev-Poincaré-Wirtinger's inequalities, and for this the flow index must satisfies $1<r<2$.

The structure of the paper is as follows. In Section 2 we introduce the domain and give the main result (Theorem 2.1). In Section 3, we establish some a priori estimates and in Section 4 we introduce the version of the unfolding method for a domain perforated by cylinders with small diameters. Some compactness results, which are the main keys when we will pass to the limit later, is addressed in Section 5. Finally, the proof of the Theorem 2.1 is given in Section 6 .

\section{Setting of the problem and main result}

Geometrical setting. A periodic porous medium is defined by a domain $\omega$ and an associated microstructure, or periodic cell $Y^{\prime}=[-1 / 2,1 / 2]^{2}$, which is made of two complementary parts: the fluid part $Y_{f}^{\prime}$, and the solid part $Y_{s}^{\prime}$, such that $Y_{f}^{\prime} \cup Y_{s}^{\prime}=Y^{\prime}$ and $Y_{f}^{\prime} \cap Y_{s}^{\prime}=\emptyset$. More precisely, we assume that $\omega$ is a smooth, bounded, connected set in $\mathbb{R}^{2}$, and that $Y_{s}^{\prime}$ is an open connected subset of $Y^{\prime}$ with a smooth boundary $\partial Y_{s}^{\prime}$, such that $\bar{Y}_{s}^{\prime}$ is strictly included in $Y^{\prime}$. We denote $Y=Y^{\prime} \times(0,1) \subset \mathbb{R}^{3}$ and so $Y_{f}=Y_{f}^{\prime} \times(0,1)$ and $Y_{s}=Y_{s}^{\prime} \times(0,1)$.

Let $\varepsilon$ and $\delta$ be positive parameters, smaller than one, where $\delta=\delta(\varepsilon)$ is such that $\delta \rightarrow 0$ as $\varepsilon \rightarrow 0$. Using both parameters, we define the parameter

$$
\sigma_{\varepsilon}=\frac{\varepsilon}{\delta^{\frac{2-r}{r}}} .
$$

We consider a thin porous medium $\Omega_{\varepsilon \delta}$ of thickness $\sigma_{\varepsilon}$ which is perforated by solid cylinders with diameter of size $\varepsilon \delta$ and distributed periodically with period $\varepsilon$. The choice of the thickness is justified by the Poincaré-Korn inequality, see Remarks 3.2 and 3.3. In addition, to work within the thin domain framework, we assume

$$
\lim _{\varepsilon \rightarrow 0} \sigma_{\varepsilon}=0 .
$$

To define the microstructure of the domain $\omega$, set

$$
Y_{\delta f}^{\prime}=Y^{\prime} \backslash \delta \bar{Y}_{s}^{\prime}
$$

such that the domain $\omega$ is covered by a regular mesh of size $\varepsilon$, i.e. for $k^{\prime} \in \mathbb{Z}^{2}$, each cell $Y_{k^{\prime}, \varepsilon}^{\prime}=\varepsilon k^{\prime}+\varepsilon Y^{\prime}$ is divided in a fluid part $Y_{\delta f_{k^{\prime}}, \varepsilon}^{\prime}$ and a solid part $Y_{\delta s_{k^{\prime}}, \varepsilon}^{\prime}$, where $Y_{\delta s_{k^{\prime}}, \varepsilon}^{\prime}$ denotes the complement in $Y_{k^{\prime}, \varepsilon}^{\prime}$ of the set $Y_{\delta f_{k^{\prime}}, \varepsilon}^{\prime}$. We observe that $Y_{k^{\prime}, \varepsilon}^{\prime}$ is similar to the unit cell $Y^{\prime}$ rescaled to size $\varepsilon$.

Thus $Y$ is divided in a fluid part $Y_{\delta f}$ and a solid part $Y_{\delta s}$, and consequently $Y_{k^{\prime}, \varepsilon}=Y_{k^{\prime}, \varepsilon}^{\prime} \times(0,1) \subset \mathbb{R}^{3}$, which is also divided in a fluid part $Y_{\delta f_{k^{\prime}}, \varepsilon}$ and a solid part $Y_{\delta s_{k^{\prime}}, \varepsilon}$. 

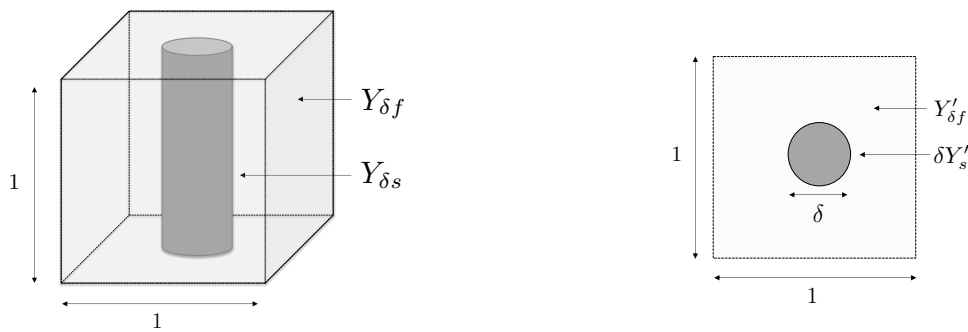

Figure 1: View of the 3D reference cells $Y$ (left) and the 2D reference cell $Y^{\prime}$ (right).

We denote by $\tau\left(\bar{Y}_{\delta s_{k^{\prime}}, \varepsilon}^{\prime}\right)$ the set of all translated images of $\bar{Y}_{\delta s_{k^{\prime}}, \varepsilon}^{\prime}$. The set $\tau\left(\bar{Y}_{\delta s_{k^{\prime}}, \varepsilon}^{\prime}\right)$ represents the solids in $\mathbb{R}^{2}$. The fluid part of the bottom $\omega_{\varepsilon \delta} \subset \mathbb{R}^{2}$ of the porous medium is defined by $\omega_{\varepsilon \delta}=\omega \backslash \bigcup_{k^{\prime} \in \mathcal{K}_{\varepsilon}} \bar{Y}_{\delta s_{k^{\prime}}, \varepsilon}^{\prime}$, where $\mathcal{K}_{\varepsilon}=\left\{k^{\prime} \in \mathbb{Z}^{2}: Y_{k^{\prime}, \varepsilon}^{\prime} \cap \omega \neq \emptyset\right\}$. Thus, the whole fluid part $\Omega_{\varepsilon \delta} \subset \mathbb{R}^{3}$ in the thin porous medium is defined by

$$
\Omega_{\varepsilon \delta}=\left\{\left(x^{\prime}, x_{3}\right) \in \mathbb{R}^{2} \times \mathbb{R}: x^{\prime} \in \omega_{\varepsilon \delta}, 0<x_{3}<\sigma_{\varepsilon}\right\} .
$$

We make the assumption that the solids $\tau\left(\bar{Y}_{\delta s_{k^{\prime}}, \varepsilon}^{\prime}\right)$ do not intersect the boundary $\partial \omega$. We define $Y_{\delta s_{k^{\prime}}, \varepsilon}^{\sigma_{\varepsilon}}=$ $Y_{\delta s_{k^{\prime}, \varepsilon}}^{\prime} \times\left(0, \sigma_{\varepsilon}\right)$. Denote by $S_{\varepsilon \delta}$ the set of the solids contained in $\Omega_{\varepsilon \delta}$. Then, $S_{\varepsilon \delta}$ is a finite union of solids, i.e. $S_{\varepsilon \delta}=\bigcup_{k^{\prime} \in \mathcal{K}_{\varepsilon}} \bar{Y}_{\delta s_{k^{\prime}}, \varepsilon}^{\sigma_{\varepsilon}}$.
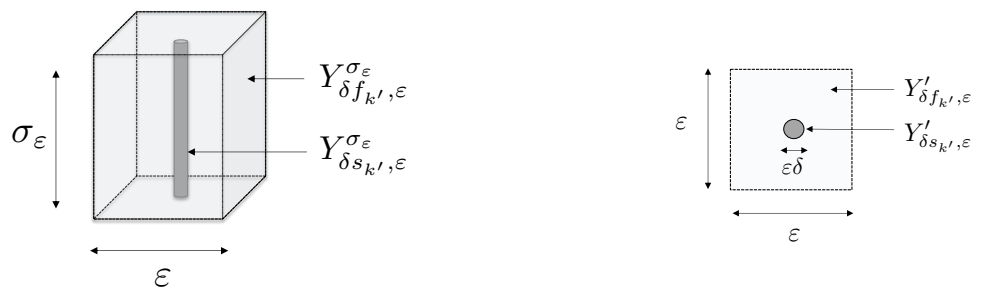

Figure 2: View of the 3D rescaled cell $Y_{k^{\prime}, \varepsilon}$ (left) and the $2 \mathrm{D}$ rescaled cell $Y_{k^{\prime}, \varepsilon}^{\prime}$ (right).

We define $\widetilde{\Omega}_{\varepsilon \delta}=\omega_{\varepsilon \delta} \times(0,1), \Omega=\omega \times(0,1)$, and $Q_{\varepsilon \delta}=\omega \times\left(0, \sigma_{\varepsilon}\right)$. We observe that $\widetilde{\Omega}_{\varepsilon \delta}=\Omega \backslash \bigcup_{k^{\prime} \in \mathcal{K}_{\varepsilon}} \bar{Y}_{\delta s_{k^{\prime}}, \varepsilon}$, and we define $T_{\varepsilon \delta}=\bigcup_{k^{\prime} \in \mathcal{K}_{\varepsilon}} \bar{Y}_{\delta s_{k^{\prime}}, \varepsilon}$ as the set of the solids contained in $\widetilde{\Omega}_{\varepsilon \delta}$.
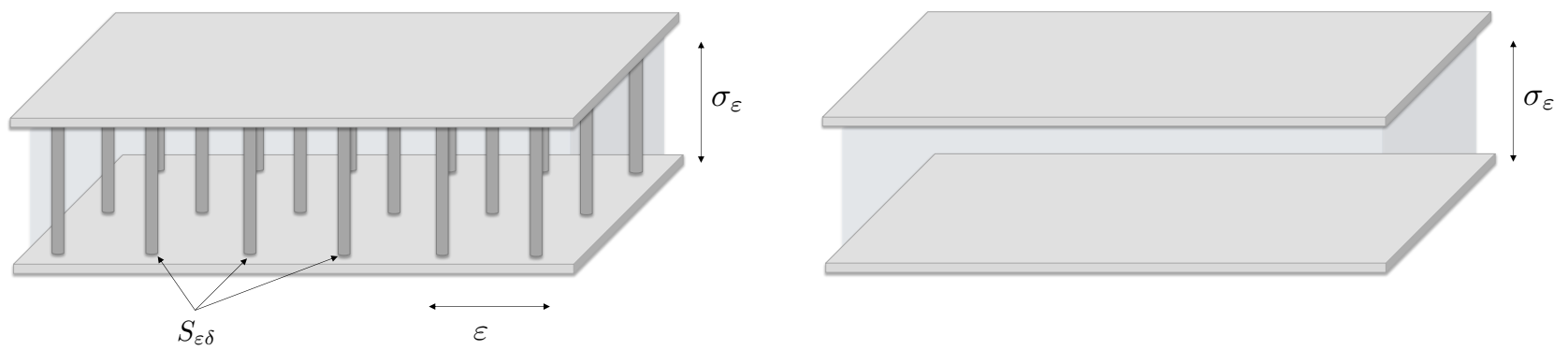

Figure 3: View of the thin porous medium $\Omega_{\varepsilon \delta}$ (left) and the domain without microstructure $Q_{\varepsilon \delta}$ (right).

Following previous notation, we remark that along the paper, the points $x \in \mathbb{R}^{3}$ will be decomposed as $x=\left(x^{\prime}, x_{3}\right)$ with $x^{\prime}=\left(x_{1}, x_{2}\right) \in \mathbb{R}^{2}, x_{3} \in \mathbb{R}$. We also use the notation $x^{\prime}$ to denote a generic vector of $\mathbb{R}^{2}$. 
Setting of the problem. In the thin porous medium $\Omega_{\varepsilon \delta}$ described above we consider the following power law Stokes system

$$
\left\{\begin{aligned}
-\mu \operatorname{div}\left(\left|\mathbb{D}\left[u_{\varepsilon \delta}\right]\right|^{r-2} \mathbb{D}\left[u_{\varepsilon \delta}\right]\right)+\nabla p_{\varepsilon \delta} & =f \text { in } \Omega_{\varepsilon \delta}, \\
\operatorname{div} u_{\varepsilon \delta} & =0 \text { in } \Omega_{\varepsilon \delta}, \\
u_{\varepsilon \delta} & =0 \text { on } \partial \Omega_{\varepsilon \delta},
\end{aligned}\right.
$$

where $u_{\varepsilon \delta}$ is the velocity field and $p_{\varepsilon \delta}$ is the pressure. In system (4), we suppose that the right-hand side (body forces) $f$ is of the form

$$
f(x)=\left(f^{\prime}\left(x^{\prime}\right), 0\right) \text {, a.e. } x \in \Omega,
$$

where $f$ is assumed in $L^{r^{\prime}}(\omega)^{2}$, where $r^{\prime}=r /(r-1)$ is the conjugate exponent of $r$. We remark that the choice of $f$ is usual when we deal with thin domains. Since the thickness of the domain is small, then the vertical component of $f$ can be neglected and moreover, it can be considered independent of the vertical variable.

Therefore, the classical theory gives the existence of a unique solution $\left(u_{\varepsilon \delta}, p_{\varepsilon \delta}\right) \in W_{0}^{1, r}\left(\Omega_{\varepsilon \delta}\right)^{3} \times L^{r^{\prime}}\left(\Omega_{\varepsilon \delta}\right)$ with $1<r<+\infty$, see Lions [17]. This solution is unique up to an additive constant for $p_{\varepsilon \delta}$, i.e. it is unique if we consider the corresponding equivalence class $p_{\varepsilon \delta} \in L^{r^{\prime}}\left(\Omega_{\varepsilon \delta}\right) / \mathbb{R}$.

Our goal is to study the asymptotic behavior of $u_{\varepsilon \delta}$ and $p_{\varepsilon \delta}$ when $\varepsilon$ and $\delta$ tend to zero. For this purpose, we use the dilatation in the variable $x_{3}$

$$
y_{3}=\frac{x_{3}}{\sigma_{\varepsilon}},
$$

in order to have the functions defined in the open set with fixed height $\widetilde{\Omega}_{\varepsilon \delta}$. Namely, we define $\tilde{u}_{\varepsilon \delta} \in W_{0}^{1, r}\left(\widetilde{\Omega}_{\varepsilon \delta}\right)^{3}$, $\tilde{p}_{\varepsilon \delta} \in L^{r^{\prime}}\left(\widetilde{\Omega}_{\varepsilon \delta}\right) / \mathbb{R}$ by

$$
\tilde{u}_{\varepsilon \delta}\left(x^{\prime}, y_{3}\right)=u_{\varepsilon \delta}\left(x^{\prime}, \sigma_{\varepsilon} y_{3}\right), \quad \tilde{p}_{\varepsilon \delta}\left(x^{\prime}, y_{3}\right)=p_{\varepsilon \delta}\left(x^{\prime}, \sigma_{\varepsilon} y_{3}\right), \text { a.e. }\left(x^{\prime}, y_{3}\right) \in \widetilde{\Omega}_{\varepsilon \delta} .
$$

Let us introduce some notation which will be useful in the following. For a vectorial function $v=\left(v^{\prime}, v_{3}\right)$ and a scalar function $w$, we will denote $\mathbb{D}_{x^{\prime}}[v]=\frac{1}{2}\left(D_{x^{\prime}} v+D_{x^{\prime}}^{t} v\right)$ and $\partial_{y_{3}}[v]=\frac{1}{2}\left(\partial_{y_{3}} v+\partial_{y_{3}}^{t} v\right)$, where we denote $\partial_{y_{3}}=\left(0,0, \frac{\partial}{\partial y_{3}}\right)^{t}$. Moreover, associated to the change of variables (5), we introduce the operators: $\mathbb{D}_{\sigma_{\varepsilon}}, D_{\sigma_{\varepsilon}}$, $\operatorname{div}_{\sigma_{\varepsilon}}$ and $\nabla_{\sigma_{\varepsilon}}$, by

$$
\begin{gathered}
\mathbb{D}_{\sigma_{\varepsilon}}[v]=\frac{1}{2}\left(D_{\sigma_{\varepsilon}} v+D_{\sigma_{\varepsilon}}^{t} v\right) \\
\left(D_{\sigma_{\varepsilon}} v\right)_{i, j}=\partial_{x_{j}} v_{i} \text { for } i=1,2,3, j=1,2, \quad\left(D_{\sigma_{\varepsilon}} v\right)_{i, 3}=\sigma_{\varepsilon}^{-1} \partial_{y_{3}} v_{i} \text { for } i=1,2,3, \\
\operatorname{div}_{\sigma_{\varepsilon}} v=\operatorname{div}_{x^{\prime}} v^{\prime}+\sigma_{\varepsilon}^{-1} \partial_{y_{3}} v_{3}, \quad \nabla_{\sigma_{\varepsilon}} w=\left(\nabla_{x^{\prime}} w, \sigma_{\varepsilon}^{-1} \partial_{y_{3}} w\right)^{t} .
\end{gathered}
$$

Using the transformation (5), system (4) can be rewritten as

$$
\left\{\begin{aligned}
-\mu \operatorname{div}_{\sigma_{\varepsilon}}\left(\left|\mathbb{D}_{\sigma_{\varepsilon}}\left[\tilde{u}_{\varepsilon \delta}\right]\right|^{p-2} \mathbb{D}_{\sigma_{\varepsilon}}\left[\tilde{u}_{\varepsilon \delta}\right]\right)+\nabla_{\sigma_{\varepsilon}} \tilde{p}_{\varepsilon \delta} & =f \text { in } \widetilde{\Omega}_{\varepsilon \delta}, \\
\operatorname{div}_{\sigma_{\varepsilon}} \tilde{u}_{\varepsilon \delta} & =0 \text { in } \widetilde{\Omega}_{\varepsilon \delta}, \\
\tilde{u}_{\varepsilon \delta} & =0 \text { on } \partial \widetilde{\Omega}_{\varepsilon \delta} .
\end{aligned}\right.
$$

Our goal then is to describe the asymptotic behavior of the dilated sequence $\left(\tilde{u}_{\varepsilon \delta}, \tilde{p}_{\varepsilon \delta}\right)$, which is given by the theorem below. However, the sequence of solutions $\left(\tilde{u}_{\varepsilon \delta}, \tilde{p}_{\varepsilon \delta}\right) \in W_{0}^{1, r}\left(\widetilde{\Omega}_{\varepsilon \delta}\right)^{3} \times L^{r^{\prime}}\left(\widetilde{\Omega}_{\varepsilon \delta}\right) / \mathbb{R}$ of system (6) is not defined in a fixed domain independent of $\varepsilon$ and $\delta$, but rather in a varying set $\widetilde{\Omega}_{\varepsilon \delta}$. Thus, in order to pass to the limit when $\varepsilon$ and $\delta$ tend to zero, convergences in fixed Sobolev spaces (defined in $\Omega$ ) are used. This previously requires to define an extension of $\left(\tilde{u}_{\varepsilon \delta}, \tilde{p}_{\varepsilon \delta}\right)$ to the whole domain $\Omega$. In this sense, we consider the zero extensions to the whole $\Omega$ for both velocity and pressure, which coincide with the original functions in $\widetilde{\Omega}_{\varepsilon \delta}$. For simplicity the extensions will be denoted by the same symbol.

Moreover, as noted in the introduction, we restrict the main result to shear thinning power law fluids. More precisely, this restriction of the flow index $r$ comes from the derivation of the Poincaré-Korn inequality (Remark 3.2) and the Sobolev-Poincaré-Wirtinger inequality (Theorem 4.3-3), which are only valid for $1<r<2$ and are necessary to obtain optimal a priori estimates of the solution. 
Theorem 2.1 (Main Theorem). Suppose $1<r<2$ and let $\sigma_{\varepsilon}$ be given by (1) satisfying (2). Then, there exist $u=\left(u^{\prime}, 0\right) \in W^{1, r}\left(0,1 ; L^{r}(\omega)^{3}\right)$ with $u=0$ on $y_{3}=\{0,1\}$ and $p \in L^{r^{\prime}}(\omega)$ independent of $y_{3}$, such that the extension $\left(\tilde{u}_{\varepsilon \delta}, \tilde{p}_{\varepsilon \delta}\right)$ of the solution of (6) satisfies the following convergences

$$
\sigma_{\varepsilon}^{-\frac{r}{r-1}} \tilde{u}_{\varepsilon \delta} \rightarrow u \quad \text { weakly in } W^{1, r}\left(0,1 ; L^{p}(\omega)^{3}\right), \quad \tilde{p}_{\varepsilon \delta} \rightarrow p \quad \text { strongly in } L^{r^{\prime}}(\Omega),
$$

where $\left(u^{\prime}, p\right)$ is the unique solution of the lower-dimensional nonlinear Brinkman type equation

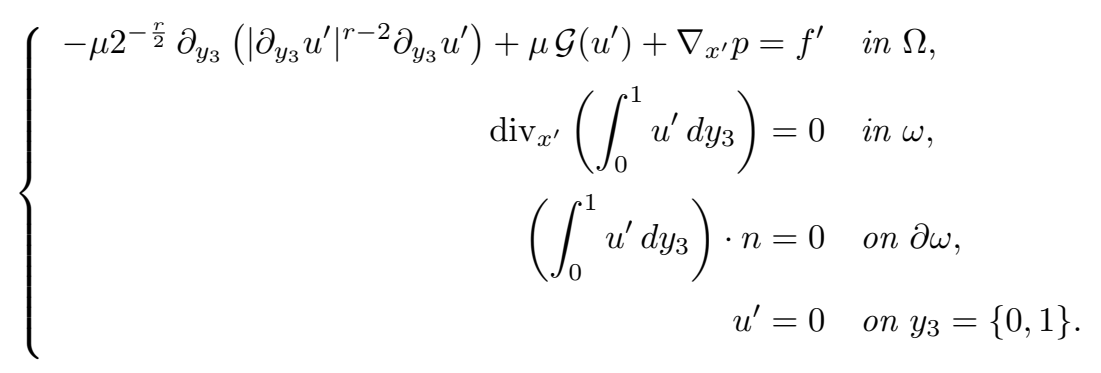

Here, the drag force function $\mathcal{G}: \mathbb{R}^{2} \rightarrow \mathbb{R}^{2}$ is defined by

$$
\mathcal{G}\left(\zeta^{\prime}\right) \cdot \tau^{\prime}=\int_{\mathbb{R}^{2} \backslash Y_{s}^{\prime}}\left|\mathbb{D}_{z^{\prime}}\left[w^{\zeta^{\prime}}\right]\right|^{r-2} \mathbb{D}_{z^{\prime}}\left[w^{\zeta^{\prime}}\right]: \mathbb{D}_{z^{\prime}}\left[w^{\tau^{\prime}}\right] d z^{\prime}, \quad \forall \tau^{\prime}, \zeta^{\prime} \in \mathbb{R}^{2},
$$

where $w^{\xi^{\prime}}, \xi^{\prime}=\tau^{\prime}, \zeta^{\prime}$, is the unique solution to the auxiliary exterior problem

$$
\left\{\begin{aligned}
-\operatorname{div}_{z^{\prime}}\left(\left|\mathbb{D}_{z^{\prime}}\left[w^{\xi^{\prime}}\right]\right|^{r-2} \mathbb{D}_{z^{\prime}}\left[w^{\xi^{\prime}}\right]\right)+\nabla_{z^{\prime}} \pi^{\xi^{\prime}}=0 & \text { in } \mathbb{R}^{2} \backslash Y_{s}^{\prime}, \\
\operatorname{div}_{z^{\prime}} w^{\xi^{\prime}}=0 & \text { in } \mathbb{R}^{2} \backslash Y_{s}^{\prime}, \\
w^{\xi^{\prime}}=0 & \text { on } \partial Y_{s}^{\prime}, \\
\lim _{\left|z^{\prime}\right| \rightarrow \infty} w^{\xi^{\prime}}=\xi^{\prime} & \\
\left(w^{\xi^{\prime}}, \pi^{\xi^{\prime}}\right) \in D^{1, r}\left(\mathbb{R}^{2} \backslash Y_{s}^{\prime}\right)^{2} \times L^{r^{\prime}}\left(\mathbb{R}^{2} \backslash Y_{s}^{\prime}\right) / \mathbb{R} &
\end{aligned}\right.
$$

The space $D^{1, r}$ denotes the homogeneous Sobolev space given by $D^{1, r}(\mathcal{O})=\left\{v \in L_{\text {loc }}^{1}(\mathcal{O}): D v \in L^{r}(\mathcal{O})\right\}$.

Remark 2.2 (Uniqueness of solution of the auxiliary exterior problem). For every $\xi^{\prime} \in \mathbb{R}^{2}$, the exterior auxiliary problem (9) has a unique solution $\left(w^{\xi^{\prime}}, \pi^{\xi^{\prime}}\right) \in D^{1, r}\left(\mathbb{R}^{2} \backslash Y_{s}^{\prime}\right)^{2} \times L^{r^{\prime}}\left(\mathbb{R}^{2} \backslash Y_{s}^{\prime}\right) / \mathbb{R}$, with $1<r<2$, see [18, Theorem 3]. For more details concerning the homogeneous Sobolev space $D^{1, r}$ we refer to [16, Chapter II.6].

Remark 2.3 (Properties of the drag force function). According to [14], the drag force function $\mathcal{G}: \mathbb{R}^{2} \rightarrow \mathbb{R}^{2}$ is continuous, strictly monotone and satisfies the homogeneity condition

$$
\mathcal{G}\left(\lambda \xi^{\prime}\right)=|\lambda|^{r-2} \lambda \mathcal{G}\left(\xi^{\prime}\right), \quad \forall\left(\lambda, \xi^{\prime}\right) \in \mathbb{R} \times \mathbb{R}^{2} .
$$

Moreover, there exists $m, M>0$ such that for every $\xi^{\prime} \in \mathbb{R}^{2}$ it holds

$$
m\left|\xi^{\prime}\right|^{r-1} \leq\left|\mathcal{G}\left(\xi^{\prime}\right)\right| \leq M\left|\xi^{\prime}\right|^{r-1} .
$$

Remark 2.4. The mathematical derivation of lower-dimensional models describing power law fluid flows in a thin domain $\Omega_{\varepsilon}=\omega \times(0, \varepsilon) \subset \mathbb{R}^{3}$ has been considered in [19], see also [3, 6, 7, 22], obtaining the nonlinear Reynolds' law

$$
\left\{\begin{array}{l}
u^{\prime}=\frac{1}{2^{\frac{r^{\prime}}{2}}\left(r^{\prime}+1\right) \mu^{r^{\prime}-1}}\left|f^{\prime}-\nabla_{x^{\prime}} p\right|^{r^{\prime}-2}\left(f^{\prime}-\nabla_{x^{\prime}} p\right) \quad \text { in } \omega \\
\operatorname{div}_{x^{\prime}} u^{\prime}=0 \text { in } \omega, \quad u^{\prime} \cdot n=0 \quad \text { on } \partial \omega
\end{array}\right.
$$


The main result of this paper can be considered a generalization of previous result to the case of a thin porous medium. In fact, if we assume the drag force function $\mathcal{G}=0$ in the Brinkman's law (7), i.e. in the absence of obstacles, we recover the homogenized model obtained in [19, Propositions 3.1 and 3.2] given by

$$
\left\{\begin{array}{l}
-\mu 2^{-\frac{r}{2}} \partial_{x_{3}}\left(\left|\partial_{x_{3}} u^{\prime}\right|^{r-2} \partial_{x_{3}} u^{\prime}\right)+\nabla_{x^{\prime}} p=f^{\prime}, \quad u_{3}=0 \quad \text { in } \Omega=\omega \times(0,1), \\
\operatorname{div}_{x^{\prime}}\left(\int_{0}^{1} u^{\prime} d x_{3}\right)=0 \quad \text { in } \omega, \quad\left(\int_{0}^{1} u^{\prime} d x_{3}\right) \cdot n=0 \quad \text { on } \partial \omega, \\
u^{\prime}=0 \quad \text { on } y_{3}=\{0,1\},
\end{array}\right.
$$

which gives rise to the nonlinear Reynolds' law (10), see [19, Propositions 3.4].

\section{A Priori Estimates}

We need some technical results to obtain a priori estimates of the solution $\left(u_{\varepsilon \delta}, p_{\varepsilon \delta}\right)$ of problem (4). First, to estimate the velocity we need a variant of the Poincaré and Korn inequalities in the thin porous medium necessary to derive uniform estimates for velocity $u_{\varepsilon \delta}$. Next, to estimate the pressure we need a result of the inverse of the divergence operator in thin porous medium.

Lemma 3.1. Suppose $1<r<+\infty$ and let $\sigma_{\varepsilon}$ be given by (1). Then, there exists a constant $C$ independent of $\varepsilon$ such that for any function $\varphi \in W_{0}^{1, r}\left(\Omega_{\varepsilon \delta}\right)^{3}$, one has

$$
\begin{array}{cl}
\|\varphi\|_{L^{r}\left(\Omega_{\varepsilon \delta}\right)^{3}} \leq C \sigma_{\varepsilon}\|D \varphi\|_{L^{r}\left(\Omega_{\varepsilon \delta}\right)^{3 \times 3}}, & 1<r<2, \quad \text { (Poincaré's inequality), } \\
\|D \varphi\|_{L^{r}\left(\Omega_{\varepsilon \delta}\right)^{3 \times 3}} \leq C\|\mathbb{D}[\varphi]\|_{L^{r}\left(\Omega_{\varepsilon \delta}\right)^{3 \times 3}}, & 1<r<+\infty, \quad \text { (Korn's inequality). }
\end{array}
$$

Proof. From [15, Lemma 4.1] applied to domain $\omega_{\varepsilon \delta}$, we have the Poincaré inequality

$$
\|\varphi\|_{L^{r}\left(\omega_{\varepsilon \delta}\right)^{3}}^{r} \leq C \sigma_{\varepsilon}^{r}\left\|D_{x^{\prime}} \varphi\right\|_{L^{r}\left(\omega_{\varepsilon \delta}\right)^{3 \times 2}}^{r} \leq C \sigma_{\varepsilon}^{r}\|D \varphi\|_{L^{r}\left(\omega_{\varepsilon \delta}\right)^{3 \times 3}}^{r}, \quad 1<r<2 .
$$

The desired result (11) follows by integrating previous estimates with respect to $x_{3}$ between 0 and $\sigma_{\varepsilon}$. Finally, the classical Korn inequality in porous mediums implies that (12) holds for $1<r<+\infty$.

Remark 3.2 (Poincaré-Korn's inequality in $\Omega_{\varepsilon \delta}$ ). Estimates given in Lemma 3.1 can be expressed as follows

$$
\|\varphi\|_{L^{r}\left(\Omega_{\varepsilon \delta}\right)^{3}} \leq C \sigma_{\varepsilon}\|\mathbb{D}[\varphi]\|_{L^{r}\left(\Omega_{\varepsilon \delta}\right)^{3 \times 3}}, \quad \forall \varphi \in W_{0}^{1, r}\left(\Omega_{\varepsilon \delta}\right)^{3}, 1<r<2 .
$$

Remark 3.3. As said in the introduction, the thin porous medium $\Omega_{\varepsilon \delta}$ is generally described by three parameters. Thus, if $\varepsilon$ and $\delta$ describe the perforation of the domain, a new parameter $h_{\varepsilon}$ should be introduced describing the thickness of the domain. In that case, it can be proved that the optimal Poincaré-Korn inequality (13) depends on the relation between $h_{\varepsilon}$ and $\sigma_{\varepsilon}$. More precisely, it can be proved that if $h_{\varepsilon} \gg \sigma_{\varepsilon}$ or $h_{\varepsilon} \approx \sigma_{\varepsilon}$ the Poincaré-Korn inequality holds with $C \sigma_{\varepsilon}$, while if $h_{\varepsilon} \ll \sigma_{\varepsilon}$ it holds with $C h_{\varepsilon}$. Therefore, the thickness of the domain considered in this paper can be viewed as the critical size of the periodic perforation with respect to the thickness of the domain, similarly to what happens in [4, Lemma 4.1].

Lemma 3.4 (Inverse of the divergence operator in $\Omega_{\varepsilon \delta}$ ). Suppose $1<r<+\infty$ and let $\sigma_{\varepsilon}$ be given by (1). Then, there exists a constant $C$ independent of $\varepsilon$ such that for any $g \in L^{r}\left(\Omega_{\varepsilon \delta}\right)$ there exists $\varphi=\varphi(g) \in W^{1, r}\left(\Omega_{\varepsilon \delta}\right)^{3}$ with $\varphi=0$ on $\partial Q_{\varepsilon \delta}$ such that

$$
\begin{gathered}
\operatorname{div} \varphi=g \text { in } \Omega_{\varepsilon \delta}, \\
\|\varphi\|_{L^{r}\left(\Omega_{\varepsilon \delta}\right)^{3}} \leq C\|g\|_{L^{r}\left(\Omega_{\varepsilon \delta}\right)}, \quad\|D \varphi\|_{L^{r}\left(\Omega_{\varepsilon \delta}\right)^{3 \times 3}} \leq C \sigma_{\varepsilon}^{-1}\|g\|_{L^{r}\left(\Omega_{\varepsilon \delta}\right)} .
\end{gathered}
$$


Proof. Let $g \in L^{r}\left(\Omega_{\varepsilon \delta}\right)$ be given. We extend $g$ inside the cylinders by means of the function:

$$
G(x)=\left\{\begin{array}{cl}
g(x) & \text { if } \quad x \in \Omega_{\varepsilon \delta}, \\
-\frac{1}{\left|Q_{\varepsilon \delta} \backslash \Omega_{\varepsilon \delta}\right|} \int_{\Omega_{\varepsilon \delta}} g(x) d x & \text { if } \quad x \in Q_{\varepsilon \delta} \backslash \Omega_{\varepsilon \delta} .
\end{array}\right.
$$

It follows that $G \in L_{0}^{r}\left(Q_{\varepsilon \delta}\right)=\left\{q \in L^{r}\left(Q_{\varepsilon \delta}\right): \int_{Q_{\varepsilon \delta}} q d x=0\right\}$ and

$$
\|G\|_{L^{r}\left(Q_{\varepsilon \delta}\right)}^{r}=\|g\|_{L^{r}\left(\Omega_{\varepsilon \delta}\right)}^{r}+\frac{1}{\left|Q_{\varepsilon \delta} \backslash \Omega_{\varepsilon \delta}\right|^{r-1}}\left|\int_{\Omega_{\varepsilon \delta}} g(x) d x\right|^{r} .
$$

Since $\left|Q_{\varepsilon \delta} \backslash \Omega_{\varepsilon \delta}\right|$ is bounded from below by a positive number, it follows from (16) and Hölder's inequality that

$$
\|G\|_{L^{r}\left(Q_{\varepsilon \delta}\right)} \leq C\|g\|_{L^{r}\left(\Omega_{\varepsilon \delta}\right)} .
$$

Besides that, since $G \in L^{r}\left(Q_{\varepsilon \delta}\right)$, it follows from [12, Lemma 4] that we can find $\varphi \in W_{0}^{1, r}\left(Q_{\varepsilon \delta}\right)^{3}$ such that

$$
\begin{gathered}
\operatorname{div} \varphi=G \text { in } Q_{\varepsilon \delta}, \\
\|\varphi\|_{L^{r}\left(Q_{\varepsilon \delta}\right)^{3}} \leq C\|G\|_{L^{r}\left(Q_{\varepsilon \delta}\right)}, \quad\|D \varphi\|_{L^{r}\left(Q_{\varepsilon \delta}\right)^{3 \times 3}} \leq C \sigma_{\varepsilon}^{-1}\|G\|_{L^{r}\left(Q_{\varepsilon \delta}\right)} .
\end{gathered}
$$

Let us consider $\varphi_{\left.\right|_{\Omega_{\varepsilon \delta}}}$ : it belongs to $W^{1, r}\left(\Omega_{\varepsilon \delta}\right)$ with $\varphi=0$ on $\partial Q_{\varepsilon \delta}$. Moreover, (14) follows from (18) and estimates given in (15) follow from (17) and (19).

By means of previous technical results, we give the estimates of the solution $\left(u_{\varepsilon \delta}, p_{\varepsilon \delta}\right)$ of problem $(4)$ in $\Omega_{\varepsilon \delta}$.

Lemma 3.5 (Estimates of velocity and pressure in $\Omega_{\varepsilon \delta}$ ). Suppose $1<r<2$ and let $\sigma_{\varepsilon}$ be given by (1). Then, there exists a constant $C$ independent of $\varepsilon$, such that if $\left(u_{\varepsilon \delta}, p_{\varepsilon \delta}\right) \in W_{0}^{1, r}\left(\Omega_{\varepsilon \delta}\right)^{3} \times L^{r^{\prime}}\left(\Omega_{\varepsilon \delta}\right) / \mathbb{R}$ is the solution of the problem (4), one has

$$
\begin{gathered}
\left\|u_{\varepsilon \delta}\right\|_{L^{r}\left(\Omega_{\varepsilon \delta}\right)^{3}} \leq C \sigma_{\varepsilon}^{\frac{2 r-1}{r(r-1)}+1}, \quad\left\|\mathbb{D}\left[u_{\varepsilon \delta}\right]\right\|_{L^{r}\left(\Omega_{\varepsilon \delta}\right)^{3 \times 3}} \leq C \sigma_{\varepsilon}^{\frac{2 r-1}{r(r-1)}} \\
\left\|D u_{\varepsilon \delta}\right\|_{L^{r}\left(\Omega_{\varepsilon \delta}\right)^{3 \times 3}} \leq C \sigma_{\varepsilon}^{\frac{2 r-1}{r(r-1)}} \\
\left\|p_{\varepsilon \delta}\right\|_{L^{r^{\prime}}\left(\Omega_{\varepsilon \delta}\right)} \leq C \sigma_{\varepsilon}^{\frac{1}{r^{\prime}}}
\end{gathered}
$$

where $r^{\prime}=\frac{r}{r-1}$.

Proof. We start with the velocity. Multiplying by $u_{\varepsilon \delta}$ in the first equation of (4) and integrating over $\Omega_{\varepsilon \delta}$, we have

$$
\mu \int_{\Omega_{\varepsilon \delta}}\left|\mathbb{D}\left[u_{\varepsilon \delta}\right]\right|^{r-2} \mathbb{D}\left[u_{\varepsilon \delta}\right]: \mathbb{D}\left[u_{\varepsilon \delta}\right] d x=\int_{\Omega_{\varepsilon \delta}} f \cdot u_{\varepsilon \delta} d x .
$$

Using Hölder's inequality and the assumption of $f$, we obtain that

$$
\int_{\Omega_{\varepsilon \delta}} f \cdot u_{\varepsilon \delta} d x^{\prime} d y_{3} \leq C \sigma_{\varepsilon}^{\frac{r-1}{r}}\left\|u_{\varepsilon \delta}\right\|_{L^{r}\left(\Omega_{\varepsilon \delta}\right)^{3}},
$$

and by (23), we have

$$
\left\|\mathbb{D}\left[u_{\varepsilon \delta}\right]\right\|_{L^{r}\left(\Omega_{\varepsilon \delta}\right)^{3 \times 3}}^{r} \leq C \sigma_{\varepsilon}^{\frac{r-1}{r}}\left\|u_{\varepsilon \delta}\right\|_{L^{r}\left(\Omega_{\varepsilon \delta}\right)^{3}} .
$$

Taking into account (13), we obtain the second estimate in (20) and consequently, from Korn's inequality (12) we obtain (21). Now, from (13) and the second estimate in (20), we deduce the first estimate in (20). 
Now, we obtain the estimate for the pressure. Let $g \in L^{r}\left(\Omega_{\varepsilon \delta}\right)$ be given. From Lemma 3.4, there exists $\varphi \in W^{1, r}\left(\Omega_{\varepsilon \delta}\right)^{3}$ with $\varphi=0$ on $\partial Q_{\varepsilon \delta}$ such that

$$
\operatorname{div} \varphi=g \text { in } \Omega_{\varepsilon \delta}, \quad\|\varphi\|_{L^{r}\left(\Omega_{\varepsilon \delta}\right)^{3}} \leq C\|g\|_{L^{r}\left(\Omega_{\varepsilon \delta}\right)}, \quad\|D \varphi\|_{L^{r}\left(\Omega_{\varepsilon \delta}\right)^{3 \times 3}} \leq C \sigma_{\varepsilon}^{-1}\|g\|_{L^{r}\left(\Omega_{\varepsilon \delta}\right)} .
$$

Multiplying by $\varphi \in W^{1, r}\left(\Omega_{\varepsilon \delta}\right)^{3}$, with $\varphi=0$ on $\partial Q_{\varepsilon \delta}$, in the first equation of (4) and integrating over $\Omega_{\varepsilon \delta}$, from the second estimate in (20) and (11), we have

$$
\left|\int_{\Omega_{\varepsilon \delta}} p_{\varepsilon \delta} \operatorname{div} \varphi d x\right| \leq C\left\|\mathbb{D}\left[u_{\varepsilon \delta}\right]\right\|_{L^{r}\left(\Omega_{\varepsilon \delta}\right)^{3 \times 3}}^{r-1}\|D \varphi\|_{L^{r}\left(\Omega_{\varepsilon \delta}\right)^{3 \times 3}}+C \sigma_{\varepsilon}^{\frac{r-1}{r}}\|\varphi\|_{L^{r}\left(\Omega_{\varepsilon \delta}\right)^{3}} \leq C \sigma_{\varepsilon}^{\frac{2 r-1}{r}}\|D \varphi\|_{L^{r}\left(\Omega_{\varepsilon \delta}\right)^{3 \times 3}},
$$

and using (25), we get

$$
\left|\int_{\Omega_{\varepsilon \delta}} p_{\varepsilon \delta} g d x\right| \leq C \sigma_{\varepsilon}^{\frac{r-1}{r}}\|g\|_{L^{r}\left(\Omega_{\varepsilon \delta}\right)} .
$$

Now, we consider in (26) the function $g=\left|p_{\varepsilon \delta}\right|^{r^{\prime}-2} p_{\varepsilon \delta}$, which satisfies $g \in L^{r}\left(\Omega_{\varepsilon \delta}\right)$ due to $p_{\varepsilon \delta} \in L^{r^{\prime}}\left(\Omega_{\varepsilon \delta}\right)$. Then, it easily follows

$$
\left\|p_{\varepsilon \delta}\right\|_{L^{r^{\prime}\left(\Omega_{\varepsilon \delta}\right)}}^{r^{\prime}} \leq C \sigma_{\varepsilon}^{\frac{r-1}{r}}\left\|p_{\varepsilon \delta}\right\|_{L^{r^{\prime}}\left(\Omega_{\varepsilon \delta}\right)}^{r^{\prime}-1}
$$

which, taking into account that $(r-1) / r=1 / r^{\prime}$, implies estimate $(22)$.

Considering the change of variables given in (5), we obtain the following result in the domain $\widetilde{\Omega}_{\varepsilon \delta}$.

Lemma 3.6 (Estimates of dilated velocity and pressure in $\left.\widetilde{\Omega}_{\varepsilon \delta}\right)$. Suppose $1<r<2$ and let $\sigma_{\varepsilon}$ be given by (1). Then, there exists a constant $C$ independent of $\varepsilon$, such that if $\left(\tilde{u}_{\varepsilon \delta}, \tilde{p}_{\varepsilon}\right) \in W_{0}^{1, r}\left(\widetilde{\Omega}_{\varepsilon \delta}\right)^{3} \times L^{r^{\prime}}\left(\widetilde{\Omega}_{\varepsilon \delta}\right) / \mathbb{R}$ is the solution of the problem (6), one has

$$
\begin{gathered}
\left\|\tilde{u}_{\varepsilon \delta}\right\|_{L^{r}\left(\widetilde{\Omega}_{\varepsilon \delta}\right)^{3}} \leq C \sigma_{\varepsilon}^{\frac{r}{r-1}}, \quad\left\|\mathbb{D}_{\sigma_{\varepsilon}}\left[\tilde{u}_{\varepsilon \delta}\right]\right\|_{L^{r}\left(\widetilde{\Omega}_{\varepsilon \delta}\right)^{3 \times 3}} \leq C \sigma_{\varepsilon}^{\frac{1}{r-1}}, \\
\left\|D_{\sigma_{\varepsilon}} \tilde{u}_{\varepsilon \delta}\right\|_{L^{r}\left(\widetilde{\Omega}_{\varepsilon \delta}\right)^{3 \times 3}} \leq C \sigma_{\varepsilon}^{\frac{1}{r-1}} \\
\left\|\tilde{p}_{\varepsilon \delta}\right\|_{L^{r^{\prime}}\left(\widetilde{\Omega}_{\varepsilon \delta}\right)} \leq C,
\end{gathered}
$$

where $r^{\prime}=\frac{r}{r-1}$.

Remark 3.7 (Extension of $\left(\tilde{u}_{\varepsilon \delta}, \tilde{p}_{\varepsilon \delta}\right)$ to the whole domain $\Omega$ ). We extend the velocity $\tilde{u}_{\varepsilon \delta}$ by zero to $\Omega$ and denote the extension by the same symbol. Obviously, estimates (27)-(28) remain valid and the extension is divergence free too. Similarly, we consider the extension by zero of the pressure $\tilde{p}_{\varepsilon \delta}$ to $\Omega$, still denoted by the same symbol, and so estimate (29) remains valid.

\section{Unfolding Method in domains with cylinders of small diameter}

The change of variable (5) does not provide the information we need about the behavior of $\left(\tilde{u}_{\varepsilon \delta}, \tilde{p}_{\varepsilon \delta}\right)$ in the microstructure associated to $\widetilde{\Omega}_{\varepsilon \delta}$. To solve this difficulty, we need to introduce an adaptation of the unfolding method adapted to perforated domains by cylinders with diameters of size $\varepsilon \delta$ distributed periodically with period $\varepsilon$, see [11, Chapter 9] for more details.

Now, let us introduce the adaption of the unfolding method in which we divide the domain $\Omega$ in cubes of lateral length $\varepsilon$ and vertical length 1 . 
Definition 4.1 (Unfolding operator in domains with cylinders of small diameter). For $\tilde{\varphi} \in L^{r}(\Omega), 1 \leq r \leq+\infty$, we define $\hat{\varphi} \in L^{r}\left(\omega \times \mathbb{R}^{2} \times(0,1)\right)$ by

$$
\hat{\varphi}\left(x^{\prime}, z^{\prime}, y_{3}\right)= \begin{cases}\tilde{\varphi}\left(\varepsilon \kappa\left(\frac{x^{\prime}}{\varepsilon}\right)+\varepsilon \delta z^{\prime}, y_{3}\right), & \text { if }\left(x^{\prime}, z^{\prime}, y_{3}\right) \in \omega \times \frac{1}{\delta} Y^{\prime} \times(0,1), \\ 0 & \text { otherwise, }\end{cases}
$$

where the function $\kappa: \mathbb{R}^{2} \rightarrow \mathbb{Z}^{2}$ is defined by

$$
\kappa\left(x^{\prime}\right)=k^{\prime} \Longleftrightarrow x^{\prime} \in Y_{k^{\prime}, 1}^{\prime}, \quad \forall k^{\prime} \in \mathbb{Z}^{2} .
$$

Remark 4.2. We make the following comments:

- For $\delta=1$ we are in presence of the adaptation of the unfolding operator for domains with cylinders introduced in [4, Subsection 4.2].

- The function $\kappa$ is well defined up to a set of zero measure in $\mathbb{R}^{2}$ (the set $\cup_{k^{\prime} \in \mathbb{Z}^{2}} \partial Y_{k^{\prime}, 1}^{\prime}$ ). Moreover, for every $\varepsilon>0$, we have

$$
\kappa\left(\frac{x^{\prime}}{\varepsilon}\right)=k^{\prime} \Longleftrightarrow x^{\prime} \in Y_{k^{\prime}, \varepsilon}^{\prime} .
$$

- For $k^{\prime} \in \mathcal{K}_{\varepsilon}$, the restriction of $\hat{\varphi}$ to $Y_{k^{\prime}, \varepsilon}^{\prime} \times \frac{1}{\delta} Y^{\prime} \times(0,1)$ does not depend on $x^{\prime}$, whereas as a function of $z^{\prime}$ it is obtained from $\tilde{\varphi}$ by using the changes of variables $\delta z^{\prime}=y^{\prime}$ and

$$
y^{\prime}=\frac{x^{\prime}-\varepsilon k^{\prime}}{\varepsilon},
$$

which transform $Y_{k^{\prime}, \varepsilon}$ into $\frac{1}{\delta} Y^{\prime} \times(0,1)$.

Next, we give some useful properties of the unfolding operator (30).

Theorem 4.3 (Properties of the unfolding operator). We have the following properties of $\hat{\varphi}$ :

1. Suppose $1 \leq r \leq+\infty$. For every $\tilde{\varphi} \in L^{r}(\Omega)$,

$$
\|\hat{\varphi}\|_{L^{r}\left(\omega \times \mathbb{R}^{2} \times(0,1)\right)} \leq \delta^{-\frac{2}{r}}\|\tilde{\varphi}\|_{L^{r}(\Omega)} .
$$

2. Suppose $1 \leq r \leq+\infty$. For every $\tilde{\varphi} \in W^{1, r}(\Omega)$,

$$
\begin{gathered}
\left\|\mathbb{D}_{z^{\prime}}[\hat{\varphi}]\right\|_{L^{r}\left(\omega \times \frac{1}{\delta} Y^{\prime} \times(0,1)\right)^{2}} \leq \sigma_{\varepsilon}\left\|\mathbb{D}_{x^{\prime}}[\tilde{\varphi}]\right\|_{L^{r}(\Omega)^{2}}, \\
\left\|\partial_{y_{3}}[\hat{\varphi}]\right\|_{L^{r}\left(\omega \times \frac{1}{\delta} Y^{\prime} \times(0,1)\right)} \leq \delta^{-\frac{2}{r}}\left\|\partial_{y_{3}}[\tilde{\varphi}]\right\|_{L^{r}(\Omega)} .
\end{gathered}
$$

3. Suppose $1 \leq r<2$ and let $\mathcal{O}$ be a bounded open set in $\mathbb{R}^{2}$. For every $\tilde{\varphi} \in W^{1, r}(\Omega)$,

$$
\begin{gathered}
\|\hat{\varphi}-\bar{\varphi}\|_{L^{r}\left(\Omega ; L^{r^{*}}\left(\mathbb{R}^{2}\right)\right)} \leq C \sigma_{\varepsilon}\left\|\mathbb{D}_{x^{\prime}}[\tilde{\varphi}]\right\|_{L^{r}(\Omega)^{2}}, \\
\|\hat{\varphi}\|_{L^{r}(\omega \times \mathcal{O} \times(0,1))} \leq C|\mathcal{O}|^{\frac{1}{2}} \sigma_{\varepsilon}\left\|\mathbb{D}_{x^{\prime}}[\tilde{\varphi}]\right\|_{L^{r}(\Omega)^{2}}+|\mathcal{O}|^{\frac{1}{r}}\|\tilde{\varphi}\|_{L^{r}(\Omega)},
\end{gathered}
$$

where $r^{*}=\frac{2 r}{2-r}$ be the associated Sobolev exponent, $C$ denotes the Sobolev-Poincaré-Wirtinger constant for $W^{1, r}\left(Y^{\prime}\right)$ and $\bar{\varphi} \in L^{r}(\Omega)$ is the local average defined by

$$
\bar{\varphi}\left(x^{\prime}, y_{3}\right)=\frac{1}{\varepsilon^{2}} \int_{\varepsilon \kappa\left(\frac{x^{\prime}}{\varepsilon}\right)+\varepsilon Y^{\prime}} \tilde{\varphi}\left(\tau^{\prime}, y_{3}\right) d \tau^{\prime}=\delta^{2} \int_{\frac{1}{\delta} Y^{\prime}} \hat{\varphi}\left(x^{\prime}, \tau^{\prime}, y_{3}\right) d \tau^{\prime}, \quad \forall \tilde{\varphi} \in L^{r}(\Omega) .
$$


Proof. 1. Using the definition (30), we have

$$
\begin{aligned}
\int_{\omega \times \mathbb{R}^{2} \times(0,1)}\left|\hat{\varphi}\left(x^{\prime}, z^{\prime}, y_{3}\right)\right|^{r} d x^{\prime} d z^{\prime} d y_{3} & \leq \sum_{k^{\prime} \in \mathcal{K}_{\varepsilon}} \int_{Y_{k^{\prime}, \varepsilon}} \int_{\mathbb{R}^{2} \times(0,1)}\left|\hat{\varphi}\left(x^{\prime}, z^{\prime}, y_{3}\right)\right|^{r} d x^{\prime} d z^{\prime} d y_{3} \\
& =\sum_{k^{\prime} \in \mathcal{K}_{\varepsilon}} \int_{Y_{k^{\prime}, \varepsilon}^{\prime}} \int_{\frac{1}{\delta} Y^{\prime} \times(0,1)}\left|\tilde{\varphi}\left(\varepsilon k^{\prime}+\varepsilon \delta z^{\prime}, y_{3}\right)\right|^{r} d x^{\prime} d z^{\prime} d y_{3} .
\end{aligned}
$$

We observe that $\tilde{\varphi}$ does not depend on $x^{\prime}$, then we obtain

$$
\int_{\omega \times \mathbb{R}^{2} \times(0,1)}\left|\hat{\varphi}\left(x^{\prime}, z^{\prime}, y_{3}\right)\right|^{r} d x^{\prime} d z^{\prime} d y_{3} \leq \varepsilon^{2} \sum_{k^{\prime} \in \mathcal{K}_{\varepsilon}} \int_{\frac{1}{\delta} Y^{\prime} \times(0,1)}\left|\tilde{\varphi}\left(\varepsilon k^{\prime}+\varepsilon \delta z^{\prime}, y_{3}\right)\right|^{r} d z^{\prime} d y_{3},
$$

and by the change of variables

$$
\delta z^{\prime}=y^{\prime}, \quad d z^{\prime}=\frac{d y^{\prime}}{\delta^{2}}, \quad \partial_{z^{\prime}}=\delta \partial_{y^{\prime}},
$$

which rescales from $\frac{1}{\delta} Y^{\prime} \times(0,1)$ to $Y$, we have

$$
\int_{\omega \times \mathbb{R}^{2} \times(0,1)}\left|\hat{\varphi}\left(x^{\prime}, z^{\prime}, y_{3}\right)\right|^{r} d x^{\prime} d z^{\prime} d y_{3} \leq \varepsilon^{2} \delta^{-2} \sum_{k^{\prime} \in \mathcal{K}_{\varepsilon}} \int_{Y}\left|\tilde{\varphi}\left(\varepsilon k^{\prime}+\varepsilon y^{\prime}, y_{3}\right)\right|^{r} d y
$$

By the change of variables (31), we have

$$
\begin{aligned}
\int_{\omega \times \mathbb{R}^{2} \times(0,1)}\left|\hat{\varphi}\left(x^{\prime}, z^{\prime}, y_{3}\right)\right|^{r} d x^{\prime} d z^{\prime} d y_{3} & \leq \delta^{-2} \sum_{k^{\prime} \in \mathcal{K}_{\varepsilon}} \int_{Y_{k^{\prime}, \varepsilon}^{\prime}} \int_{0}^{1}\left|\tilde{\varphi}\left(x^{\prime}, y_{3}\right)\right|^{r} d x^{\prime} d y_{3} \\
& \leq \delta^{-2} \int_{\omega \times(0,1)}\left|\tilde{\varphi}\left(x^{\prime}, y_{3}\right)\right|^{r} d x^{\prime} d y_{3}
\end{aligned}
$$

i.e.,

$$
\|\hat{\varphi}\|_{L^{r}\left(\omega \times \mathbb{R}^{2} \times(0,1)\right)} \leq \delta^{-\frac{2}{r}}\|\tilde{\varphi}\|_{L^{r}(\Omega)} .
$$

2. Observe that, by the definition (30), we can deduce

$$
\int_{\omega \times \frac{1}{\delta} Y^{\prime} \times(0,1)}\left|\mathbb{D}_{z^{\prime}}\left[\hat{\varphi}\left(x^{\prime}, z^{\prime}, y_{3}\right)\right]\right|^{r} d x^{\prime} d z^{\prime} d y_{3} \leq \sum_{k^{\prime} \in \mathcal{K}_{\varepsilon}} \int_{Y_{k^{\prime}, \varepsilon}^{\prime}} \int_{\frac{1}{\delta} Y^{\prime} \times(0,1)}\left|\mathbb{D}_{z^{\prime}}\left[\tilde{\varphi}\left(\varepsilon k^{\prime}+\varepsilon \delta z^{\prime}, y_{3}\right)\right]\right|^{r} d x^{\prime} d z^{\prime} d y_{3} .
$$

As $\tilde{\varphi}$ does not depend on $x^{\prime}$, then we obtain

$$
\int_{\omega \times \frac{1}{\delta} Y^{\prime} \times(0,1)}\left|\mathbb{D}_{z^{\prime}}\left[\hat{\varphi}\left(x^{\prime}, z^{\prime}, y_{3}\right)\right]\right|^{r} d x^{\prime} d z^{\prime} d y_{3} \leq \varepsilon^{2} \sum_{k^{\prime} \in \mathcal{K}_{\varepsilon}} \int_{\frac{1}{\delta} Y^{\prime} \times(0,1)}\left|\mathbb{D}_{z^{\prime}}\left[\tilde{\varphi}\left(\varepsilon k^{\prime}+\varepsilon \delta z^{\prime}, y_{3}\right)\right]\right|^{r} d z^{\prime} d y_{3},
$$

and by the change of variables (38), we have

$$
\int_{\omega \times \frac{1}{\delta} Y^{\prime} \times(0,1)}\left|\mathbb{D}_{z^{\prime}}\left[\hat{\varphi}\left(x^{\prime}, z^{\prime}, y_{3}\right)\right]\right|^{r} d x^{\prime} d z^{\prime} d y_{3} \leq \varepsilon^{2} \delta^{r-2} \sum_{k^{\prime} \in \mathcal{K}_{\varepsilon}} \int_{Y}\left|\mathbb{D}_{y^{\prime}}\left[\tilde{\varphi}\left(\varepsilon k^{\prime}+\varepsilon y^{\prime}, y_{3}\right)\right]\right|^{r} d y .
$$

By the change of variables (31), we have

$$
\begin{aligned}
\int_{\omega \times \frac{1}{\delta} Y^{\prime} \times(0,1)}\left|\mathbb{D}_{z^{\prime}}\left[\hat{\varphi}\left(x^{\prime}, z^{\prime}, y_{3}\right)\right]\right|^{r} d x^{\prime} d z^{\prime} d y_{3} & \leq \varepsilon^{r} \delta^{r-2} \sum_{k^{\prime} \in \mathcal{K}_{\varepsilon}} \int_{Y_{k^{\prime}, \varepsilon}^{\prime}} \int_{0}^{1}\left|\mathbb{D}_{x^{\prime}}\left[\tilde{\varphi}\left(x^{\prime}, y_{3}\right)\right]\right|^{r} d x^{\prime} d y_{3} \\
& \leq \varepsilon^{r} \delta^{r-2} \int_{\omega \times(0,1)}\left|\mathbb{D}_{x^{\prime}}\left[\tilde{\varphi}\left(x^{\prime}, y_{3}\right)\right]\right|^{r} d x^{\prime} d y_{3},
\end{aligned}
$$


i.e.,

$$
\left\|\mathbb{D}_{z^{\prime}}[\hat{\varphi}]\right\|_{L^{r}\left(\omega \times \frac{1}{\delta} Y^{\prime} \times(0,1)\right)^{2}} \leq \varepsilon \delta \delta^{-\frac{2}{r}}\left\|\mathbb{D}_{x^{\prime}}[\tilde{\varphi}]\right\|_{L^{r}(\Omega)^{2}}=\sigma_{\varepsilon}\left\|\mathbb{D}_{x^{\prime}}[\tilde{\varphi}]\right\|_{L^{r}(\Omega)^{2}} .
$$

Similarly, using definition (30) and the change of variables (38), we have

$$
\int_{\omega \times \frac{1}{\delta} Y^{\prime} \times(0,1)}\left|\partial_{y_{3}}\left[\hat{\varphi}\left(x^{\prime}, z^{\prime}, y\right)\right]\right|^{r} d x^{\prime} d z^{\prime} d y_{3} \leq \varepsilon^{2} \delta^{-2} \sum_{k^{\prime} \in \mathcal{K}_{\varepsilon}} \int_{Y}\left|\partial_{y_{3}}\left[\tilde{\varphi}\left(\varepsilon k^{\prime}+\varepsilon y^{\prime}, y_{3}\right)\right]\right|^{r} d y .
$$

By the change of variables (31), we obtain

$$
\int_{\omega \times \frac{1}{\delta} Y^{\prime} \times(0,1)}\left|\partial_{y_{3}}\left[\hat{\varphi}\left(x^{\prime}, z^{\prime}, y_{3}\right)\right]\right|^{r} d x^{\prime} d z^{\prime} d y_{3} \leq \delta^{-2} \int_{\omega \times(0,1)}\left|\partial_{y_{3}}\left[\tilde{\varphi}\left(x^{\prime}, y_{3}\right)\right]\right|^{r} d x^{\prime} d y_{3},
$$

i.e.,

$$
\left\|\partial_{y_{3}}[\hat{\varphi}]\right\|_{L^{r}\left(\omega \times \frac{1}{\delta} Y^{\prime} \times(0,1)\right)} \leq \delta^{-\frac{2}{r}}\left\|\partial_{y_{3}}[\tilde{\varphi}]\right\|_{L^{r}(\Omega)} .
$$

3. Suposse $1 \leq r<2$. For a.e. $\left(x^{\prime}, y_{3}\right) \in \Omega$, taking into account the definition of the local average (37), we proceed as previous statements to obtain

$$
\begin{aligned}
& \|\hat{\varphi}-\bar{\varphi}\|_{L^{r}\left(\Omega ; L^{r^{*}}\left(\mathbb{R}^{2}\right)\right)}^{r}\left(\int_{k^{\prime} \in \mathcal{K}_{\varepsilon}} \int_{Y_{k^{\prime}, \varepsilon}^{\prime} \times(0,1)}\left|\tilde{\varphi}\left(\varepsilon k^{\prime}+\varepsilon \delta z^{\prime}, y_{3}\right)-\delta^{2} \int_{\frac{1}{\delta} Y^{\prime}} \tilde{\varphi}\left(\varepsilon k^{\prime}+\varepsilon \delta \tau^{\prime}, y_{3}\right) d \tau^{\prime}\right|^{r^{*}} d z^{\prime}\right)^{\frac{r}{r^{*}}} d x^{\prime} d y_{3} \\
& \leq \varepsilon^{2} \sum_{k^{\prime} \in \mathcal{K}_{\varepsilon}} \int_{0}^{1}\left(\int_{\frac{1}{\delta} Y^{\prime}}\left|\tilde{\varphi}\left(\varepsilon k^{\prime}+\varepsilon \delta z^{\prime}, y_{3}\right)-\delta^{2} \int_{\frac{1}{\delta} Y^{\prime}} \tilde{\varphi}\left(\varepsilon k^{\prime}+\varepsilon \delta \tau^{\prime}, y_{3}\right) d \tau^{\prime}\right|^{r^{*}} d z^{\prime}\right)^{\frac{r}{r^{*}}} d y_{3} .
\end{aligned}
$$

From the change of variables (38), we have

$$
\begin{aligned}
& \|\hat{\varphi}-\bar{\varphi}\|_{L^{r}\left(\Omega ; L^{r^{*}}\left(\mathbb{R}^{2}\right)\right)}^{r} \\
& \leq \varepsilon^{2} \delta^{-2 \frac{r}{r^{*}}} \sum_{k^{\prime} \in \mathcal{K}_{\varepsilon}} \int_{0}^{1}\left(\int_{Y^{\prime}}\left|\tilde{\varphi}\left(\varepsilon k^{\prime}+\varepsilon y^{\prime}, y_{3}\right)-\int_{Y^{\prime}} \tilde{\varphi}\left(\varepsilon k^{\prime}+\varepsilon \tau^{\prime}, y_{3}\right) d \tau^{\prime}\right|^{r^{*}} d y^{\prime}\right)^{\frac{r}{r^{*}}} d y_{3} .
\end{aligned}
$$

Applying the Sobolev-Poincaré-Wirtinger inequality in $W^{1, r}\left(Y^{\prime}\right)$ with $1 \leq r<2$

$$
\left(\int_{Y^{\prime}}\left|\tilde{\varphi}\left(\varepsilon k^{\prime}+\varepsilon y^{\prime}, y_{3}\right)-\int_{Y^{\prime}} \tilde{\varphi}\left(\varepsilon k^{\prime}+\varepsilon \tau^{\prime}, y_{3}\right) d \tau^{\prime}\right|^{r^{*}} d y^{\prime}\right)^{\frac{1}{r^{*}}} \leq C\left(\int_{Y^{\prime}}\left|D_{y^{\prime}} \tilde{\varphi}\left(\varepsilon k^{\prime}+\varepsilon y^{\prime}, y_{3}\right)\right|^{r} d y^{\prime}\right)^{\frac{1}{r}}, \quad \forall k^{\prime} \in \mathcal{K}_{\varepsilon},
$$

we deduce

$$
\|\hat{\varphi}-\bar{\varphi}\|_{L^{r}\left(\Omega ; L^{r^{*}}\left(\mathbb{R}^{2}\right)\right)}^{r} \leq C \varepsilon^{2} \delta^{-2 \frac{r}{r^{*}}} \sum_{k^{\prime} \in \mathcal{K}_{\varepsilon}} \int_{Y^{\prime}} \int_{0}^{1}\left|D_{y^{\prime}} \tilde{\varphi}\left(\varepsilon k^{\prime}+\varepsilon y^{\prime}, y_{3}\right)\right|^{r} d y^{\prime} d y_{3} .
$$

By using the change of variables (31), we obtain

$$
\|\hat{\varphi}-\bar{\varphi}\|_{L^{r}\left(\Omega ; L^{r^{*}}\left(\mathbb{R}^{2}\right)\right)}^{r} \leq C \varepsilon^{r} \delta^{-2 \frac{r}{r^{*}}} \sum_{k^{\prime} \in \mathcal{K}_{\varepsilon}} \int_{Y_{k^{\prime}, \varepsilon}^{\prime}} \int_{0}^{1}\left|D_{x^{\prime}} \tilde{\varphi}\left(x^{\prime}, y_{3}\right)\right|^{r} d x^{\prime} d y_{3},
$$

which from $r^{*}=\frac{2 r}{2-r}$ and Korn's inequality gives

$$
\|\hat{\varphi}-\bar{\varphi}\|_{L^{r}\left(\Omega ; L^{r^{*}}\left(\mathbb{R}^{2}\right)\right)} \leq C \varepsilon \delta^{\frac{r-2}{r}}\left\|\mathbb{D}_{x^{\prime}}[\tilde{\varphi}]\right\|_{L^{r}(\Omega)^{2}}=C \sigma_{\varepsilon}\left\|\mathbb{D}_{x^{\prime}}[\tilde{\varphi}]\right\|_{L^{r}(\Omega)^{2}} .
$$


Finally, let $\mathcal{O}$ be a bounded open set in $\mathbb{R}^{2}$. Then we have

$$
\|\hat{\varphi}\|_{L^{r}(\omega \times \mathcal{O} \times(0,1))} \leq\|\hat{\varphi}-\bar{\varphi}\|_{L^{r}(\omega \times \mathcal{O} \times(0,1))}+\|\bar{\varphi}\|_{L^{r}(\omega \times \mathcal{O} \times(0,1))} .
$$

For the first term in the right-hand side of (39), by using Hölder's inequality with $q=2 /(2-r)$ and $q^{\prime}=2 / r$, and taking into account that $r q=r^{*}$, we get

$$
\begin{aligned}
\|\hat{\varphi}-\bar{\varphi}\|_{L^{r}(\omega \times \mathcal{O} \times(0,1))} & =\left(\int_{\omega \times \mathcal{O} \times(0,1)}\left|\hat{\varphi}\left(x^{\prime}, z^{\prime}, y_{3}\right)-\bar{\varphi}\left(x^{\prime}, y_{3}\right)\right|^{r} d x^{\prime} d z^{\prime} d y_{3}\right)^{\frac{1}{r}} \\
& \leq\left(\int_{\Omega}|\mathcal{O}|^{\frac{1}{q^{\prime}}}\left(\int_{\mathcal{O}}\left|\hat{\varphi}\left(x^{\prime}, z^{\prime}, y_{3}\right)-\bar{\varphi}\left(x^{\prime}, y_{3}\right)\right|^{r q} d z^{\prime}\right)^{\frac{1}{q}} d x^{\prime} d y_{3}\right)^{\frac{1}{r}} \\
& =|\mathcal{O}|^{\frac{1}{r q^{\prime}}}\left(\int_{\Omega}\left(\int_{\mathcal{O}}\left|\hat{\varphi}\left(x^{\prime}, z^{\prime}, y_{3}\right)-\bar{\varphi}\left(x^{\prime}, y_{3}\right)\right|^{r q} d z^{\prime}\right)^{\frac{r}{r q}} d x^{\prime} d y_{3}\right)^{\frac{1}{r}} \\
& =|\mathcal{O}|^{\frac{1}{2}}\|\hat{\varphi}-\bar{\varphi}\|_{L^{r}\left(\Omega ; L^{r *}\left(\mathbb{R}^{2}\right)\right) .}
\end{aligned}
$$

For the second one, since $\bar{\varphi}$ does not depend on $z^{\prime}$, we have

$$
\|\bar{\varphi}\|_{L^{r}(\omega \times \mathcal{O} \times(0,1))}=|\mathcal{O}|^{\frac{1}{r}}\|\bar{\varphi}\|_{L^{r}(\Omega)} .
$$

By using Hölder's inequality and (32), we have

$$
\begin{aligned}
\|\bar{\varphi}\|_{L^{r}(\Omega)} & =\left(\int_{\Omega}\left|\delta^{2} \int_{\frac{1}{\delta} Y^{\prime}} \hat{\varphi}\left(x^{\prime}, \tau^{\prime}, y_{3}\right) d \tau^{\prime}\right|^{r} d x^{\prime} d y_{3}\right)^{\frac{1}{r}} \\
& \leq \delta^{2}\left(\int_{\Omega}\left|\frac{1}{\delta} Y^{\prime}\right| \frac{r}{r^{\prime}}\left(\int_{\frac{1}{\delta} Y^{\prime}}\left|\hat{\varphi}\left(x^{\prime}, \tau^{\prime}, y_{3}\right)\right|^{r} d \tau^{\prime}\right) d x^{\prime} d y_{3}\right)^{\frac{1}{r}} \\
& =\delta^{2-\frac{2}{r^{\prime}}}\|\hat{\varphi}\|_{L^{r}\left(\omega \times \mathbb{R}^{2} \times(0,1)\right)} \\
& \leq\|\tilde{\varphi}\|_{L^{r}(\Omega)},
\end{aligned}
$$

which implies

$$
\|\bar{\varphi}\|_{L^{r}(\omega \times \mathcal{O} \times(0,1))} \leq|\mathcal{O}|^{\frac{1}{r}}\|\tilde{\varphi}\|_{L^{r}(\Omega)} .
$$

Then, we have that (39) reads

$$
\|\hat{\varphi}\|_{L^{r}(\omega \times \mathcal{O} \times(0,1))} \leq|\mathcal{O}|^{\frac{1}{2}}\|\hat{\varphi}-\bar{\varphi}\|_{L^{r}\left(\Omega ; L^{r^{*}}\left(\mathbb{R}^{2}\right)\right)}+|\mathcal{O}|^{\frac{1}{r}}\|\tilde{\varphi}\|_{L^{r}(\Omega)},
$$

which together with (35) gives (36).

Now, from extensions $\left(\tilde{u}_{\varepsilon \delta}, \tilde{p}_{\varepsilon \delta}\right)$, we define $\left(\hat{u}_{\varepsilon \delta}, \hat{p}_{\varepsilon \delta}\right)$ by using $(30)$. Below, we get the estimates for this sequence.

Lemma 4.4 (Estimates of the unfolded velocity and pressure). Suppose $1<r<2$, let $\sigma_{\varepsilon}$ be given by (1) and $\mathcal{O}$ be a bounded open set in $\mathbb{R}^{2}$. Then, there exists a constant $C$ independent of $\varepsilon$, such that $\left(\hat{u}_{\varepsilon \delta}, \hat{p}_{\varepsilon \delta}\right)$, defined by (30), satisfies

$$
\begin{gathered}
\left\|\hat{u}_{\varepsilon \delta}\right\|_{L^{r}(\omega \times \mathcal{O} \times(0,1))^{3}} \leq C \sigma_{\varepsilon}^{\frac{r}{r-1}} \\
\left\|\mathbb{D}_{z^{\prime}}\left[\hat{u}_{\varepsilon \delta}\right]\right\|_{L^{r}\left(\omega \times \frac{1}{\delta} Y^{\prime} \times(0,1)\right)^{3 \times 2}} \leq C \sigma_{\varepsilon}^{\frac{r}{r-1}}, \quad\left\|\partial_{y_{3}}\left[\hat{u}_{\varepsilon \delta}\right]\right\|_{L^{r}\left(\omega \times \frac{1}{\delta} Y^{\prime} \times(0,1)\right)^{3}} \leq C \sigma_{\varepsilon}^{\frac{r}{r-1}} \delta^{-\frac{2}{r}},
\end{gathered}
$$




$$
\begin{gathered}
\left\|D_{z^{\prime}} \hat{u}_{\varepsilon \delta}\right\|_{L^{r}\left(\omega \times \frac{1}{\delta} Y^{\prime} \times(0,1)\right)^{3 \times 2}} \leq C \sigma_{\varepsilon}^{\frac{r}{r-1}}, \quad\left\|\partial_{y_{3}} \hat{u}_{\varepsilon \delta}\right\|_{L^{r}\left(\omega \times \frac{1}{\delta} Y^{\prime} \times(0,1)\right)^{3}} \leq C \sigma_{\varepsilon}^{\frac{r}{r-1}} \delta^{-\frac{2}{r}}, \\
\left\|\hat{u}_{\varepsilon \delta}-\bar{u}_{\varepsilon \delta}\right\|_{L^{r}\left(\Omega ; L^{r^{*}}\left(\mathbb{R}^{2}\right)^{3}\right)} \leq C \sigma_{\varepsilon}^{\frac{r}{r-1}} \\
\left\|\hat{p}_{\varepsilon \delta}\right\|_{L^{r^{\prime}}\left(\omega \times \mathbb{R}^{2} \times(0,1)\right) / \mathbb{R}} \leq C \delta^{-\frac{2(r-1)}{r}}
\end{gathered}
$$

Proof. Taking into account the first estimate in (27) and estimate (28) in (36), we deduce (40). We remark that if we had used (27) and (32), we would have obtained estimate $\left\|\hat{u}_{\varepsilon \delta}\right\|_{L^{r}\left(\omega \times \mathbb{R}^{2} \times(0,1)\right)^{3}} \leq C \sigma_{\varepsilon}^{\frac{r}{r-1}} \delta^{-\frac{2}{r}}$, which is not as sharp a (40). Also, taking into account the second estimate in (27) and (33), we get the first estimate in (41). And using the second estimate in (27) and (34), we get the second estimate in (41). Consequently, from Korn's inequality (12), we also have (42). Estimate (35) together with the second estimate in (27) gives (43). Finally, taking into account the estimate (29) in (32), and $r^{\prime}=r /(r-1)$, we can deduce (44).

\section{Some compactness results}

In this section, we obtain some compactness results about the behavior of the extension of the sequence $\left(\tilde{u}_{\varepsilon \delta}, \tilde{p}_{\varepsilon \delta}\right)$ and the sequence $\hat{u}_{\varepsilon \delta}$ satisfying the a priori estimates given in Lemmas 3.6 and 4.4, respectively.

Lemma 5.1 (Compactness results for extension of dilated velocity and pressure). Suppose $1<r<2$ and let $\sigma_{\varepsilon}$ be given by (1) satisfying (2). Then, for a subsequence of $\varepsilon$, still denoted by $\varepsilon$, there exist $p \in L^{r^{\prime}}(\Omega) / \mathbb{R}$ independent of $y_{3}$ and $u \in W_{0}^{1, r}\left(0,1 ; L^{r}(\omega)^{3}\right)$ with $u_{3}=0$, such that

$$
\begin{gathered}
\sigma_{\varepsilon}^{-\frac{r}{r-1}} \tilde{u}_{\varepsilon \delta} \rightarrow\left(u^{\prime}, 0\right) \quad \text { weakly in } W^{1, r}\left(0,1 ; L^{r}(\omega)^{3}\right), \\
\operatorname{div}_{x^{\prime}}\left(\int_{0}^{1} u^{\prime}\left(x^{\prime}, y_{3}\right) d y_{3}\right)=0 \quad \text { in } \omega, \quad\left(\int_{0}^{1} u^{\prime}\left(x^{\prime}, y_{3}\right) d y_{3}\right) \cdot n=0 \quad \text { on } \partial \omega, \\
\tilde{p}_{\varepsilon \delta} \rightarrow p \quad \text { strongly in } L^{r^{\prime}}(\Omega) / \mathbb{R} .
\end{gathered}
$$

Proof. First, we focus on the convergence of the extension of the velocity. The first estimate given in (27) and estimate (28) imply the existence of $u \in W^{1, r}\left(0,1 ; L^{r}(\omega)^{3}\right)$ such that, up to a subsequence, it holds

$$
\sigma_{\varepsilon}^{-\frac{r}{r-1}} \tilde{u}_{\varepsilon \delta} \rightarrow u \quad \text { weakly in } W^{1, r}\left(0,1 ; L^{r}(\omega)^{3}\right),
$$

which implies

$$
\sigma_{\varepsilon}^{-\frac{r}{r-1}} \operatorname{div}_{x^{\prime}} \tilde{u}_{\varepsilon \delta}^{\prime} \rightarrow \operatorname{div}_{x^{\prime}} u^{\prime} \quad \text { weakly in } W^{1, r}\left(0,1 ; W^{-1, r^{\prime}}(\omega)\right) .
$$

Since $\operatorname{div}_{\sigma_{\varepsilon}} \tilde{u}_{\varepsilon \delta}=0$ in $\Omega$, multiplying by $\sigma_{\varepsilon}^{-\frac{r}{r-1}}$ we obtain

$$
\sigma_{\varepsilon}^{-\frac{r}{r-1}} \operatorname{div}_{x^{\prime}} \tilde{u}_{\varepsilon \delta}^{\prime}+\sigma_{\varepsilon}^{-\frac{2 r-1}{r-1}} \partial_{y_{3}} \tilde{u}_{\varepsilon \delta, 3}=0 \quad \text { in } \Omega,
$$

which combined with (49) implies that $\sigma_{\varepsilon}^{-\frac{2 r-1}{r-1}} \partial_{y_{3}} \tilde{u}_{\varepsilon \delta, 3}$ is bounded in $L^{r}\left(0,1 ; W^{-1, r^{\prime}}(\omega)\right)$. Using then that $\tilde{u}_{\varepsilon \delta, 3}=$ 0 on $\omega \times\{1\}$, we deduce that $\sigma_{\varepsilon}^{-\frac{2 r-1}{r-1}} \tilde{u}_{\varepsilon \delta, 3}$ is bounded in $W^{1, r}\left(0,1 ; W^{-1, r^{\prime}}(\omega)\right)$, and therefore, up to a subsequence, there exists $w \in W^{1, r}\left(0,1 ; W^{-1, r^{\prime}}(\omega)\right)$ with $w(1)=0$ in $W^{-1, r^{\prime}}(\omega)$, such that

$$
\sigma_{\varepsilon}^{-\frac{2 r-1}{r-1}} \tilde{u}_{\varepsilon \delta, 3} \rightarrow w \quad \text { weakly in } W^{1, r}\left(0,1 ; W^{-1, r^{\prime}}(\omega)\right) .
$$

From this convergence and (48), we get that $\sigma_{\varepsilon}^{-\frac{r}{r-1}} \tilde{u}_{\varepsilon \delta, 3}$ tends to zero and so, by uniqueness of the limit, $u_{3}=0$ which finishes the proof of (45). Moreover, $\tilde{u}_{\varepsilon \delta}=0$ on $y_{3}=\{0,1\}$ and the continuity of the trace applications from the space of functions $u$ such that $\|u\|_{L^{r}}$ and $\left\|\partial_{y_{3}} u\right\|_{L^{r}}$ to $L^{r}\left(\omega \times\left\{y_{3}\right\}\right)$ with $y_{3}=\{0,1\}$ imply that $u=0$ on $y_{3}=\{0,1\}$ and so $u \in W_{0}^{1, r}\left(0,1 ; L^{r}(\omega)^{3}\right)$ with $u_{3}=0$. 
Next, we prove the divergence condition (46). To do this, we consider $\varphi \in C^{1}(\omega)$ as test function in $\operatorname{div}_{\sigma_{\varepsilon}} \tilde{u}_{\varepsilon \delta}=0$ in $\Omega$ and taking into account that $\tilde{u}_{\varepsilon \delta}=0$ on $\partial \Omega$, we get

$$
0=\int_{\Omega}\left(\sigma_{\varepsilon}^{-\frac{r}{r-1}} \operatorname{div}_{x^{\prime}} \tilde{u}_{\varepsilon \delta}^{\prime}+\sigma_{\varepsilon}^{-\frac{2 r-1}{r-1}} \partial_{y_{3}} \tilde{u}_{\varepsilon \delta, 3}\right) \varphi\left(x^{\prime}\right) d x^{\prime} d y_{3}=-\int_{\Omega} \sigma_{\varepsilon}^{-\frac{r}{r-1}} \tilde{u}_{\varepsilon \delta}^{\prime} \cdot \nabla_{x^{\prime}} \varphi\left(x^{\prime}\right) d x^{\prime} d y_{3} .
$$

From convergence (45), we get

$$
\int_{\Omega} u^{\prime} \cdot \nabla_{x^{\prime}} \varphi\left(x^{\prime}\right) d x^{\prime} d y_{3}=0
$$

which implies (46).

Last, we focus on the extension of the pressure by proving (47). From estimate (29) we deduce that there exists $p \in L^{r^{\prime}}(\Omega) / \mathbb{R}$ such that, up to a subsequence, it holds

$$
\tilde{p}_{\varepsilon \delta} \rightarrow p \quad \text { weakly in } L^{r^{\prime}}(\Omega) / \mathbb{R} .
$$

To prove that $p$ is independent of $y_{3}$, we multiply system (6) by $\sigma_{\varepsilon} \varphi$ with $\varphi \in C_{0}^{\infty}(\Omega)$. Integrating by parts and taking into account estimates of the extension of the velocity (27), assumption of force $f$ and convergence (51), we pass to the limit and obtain

$$
\int_{\Omega} p \partial_{y_{3}} \varphi d x^{\prime} d y_{3}=0
$$

This implies that $p$ does not depend on $y_{3}$. Moreover, if we argue similarly as in [8, Lemma 4.4], we have that the convergence of the pressure $\tilde{p}_{\varepsilon \delta}$ is in fact strong, which concludes the proof.

Next, we give a compactness result for the unfolded function $\hat{u}_{\varepsilon \delta}$. To do this, following [11, Chapters 9 and 10], we consider the homogeneous Sobolev space of weakly differentiable functions defined locally on $\mathbb{R}^{2}$ having a gradient in $L^{r}\left(\mathbb{R}^{2}\right)^{2}$ and zero value on the obstacle $Y_{s}^{\prime}$, which is given by

$$
\mathbf{K}_{Y_{s}^{\prime}}=\left\{\Phi\left(z^{\prime}\right) \in W_{l o c}^{1, r}\left(\mathbb{R}^{2}\right): \nabla_{z^{\prime}} \Phi \in L^{r}\left(\mathbb{R}^{2}\right)^{2} \text { and } \Phi=0 \text { on } Y_{s}^{\prime}\right\} .
$$

We remark that if $\Phi \in \mathbf{K}_{Y_{s}^{\prime}}$ then it has a limit at infinity denoted $\Phi_{\infty}$, i.e. there exists $\Phi_{\infty} \in \mathbb{R}$ such that $\lim _{\left|z^{\prime}\right| \rightarrow+\infty} \Phi\left(z^{\prime}\right)=\Phi_{\infty}$. In addition, to relate the value at infinity of the limit of $\hat{u}_{\varepsilon \delta}$ with the limit of $\tilde{u}_{\varepsilon \delta}$, we consider a more general space

$$
\mathbf{L}_{Y_{s}^{\prime}}=\left\{\Phi\left(x^{\prime}, z^{\prime}, y_{3}\right) \in L^{r}\left(\Omega ; \mathbf{K}_{Y_{s}^{\prime}}\right): \Phi_{\infty}=\Phi(\cdot, \infty, \cdot) \in W_{0}^{1, r}\left(0,1 ; L^{r}(\omega)\right)\right\} .
$$

Lemma 5.2 (Compactness results for unfolded velocity). Suppose $1<r<2$, let $\sigma_{\varepsilon}$ be given by (1) satisfying (2) and $u \in W_{0}^{1, r}\left(0,1 ; L^{r}(\omega)^{3}\right)$ be given in Lemma 5.1. Then, for a subsequence of $\varepsilon$, still denoted by $\varepsilon$, there exists $U \in \mathbf{L}_{Y_{s}^{\prime}}^{3}$ where $U_{\infty}=u$ and $U_{3}$ is independent of $y_{3}$, such that

$$
\begin{gathered}
\sigma_{\varepsilon}^{-\frac{r}{r-1}} \hat{u}_{\varepsilon \delta} \rightarrow U \quad \text { weakly in } L^{r}\left(\Omega ; L_{l o c}^{r}\left(\mathbb{R}^{2}\right)^{3}\right), \\
\sigma_{\varepsilon}^{-\frac{r}{r-1}} D_{z^{\prime}} \hat{u}_{\varepsilon \delta} \mathbf{1}_{\frac{1}{\delta} Y^{\prime}} \rightarrow D_{z^{\prime}} U \quad \text { weakly in } L^{r}\left(\omega \times \mathbb{R}^{2} \times(0,1)\right)^{3 \times 2}, \\
\operatorname{div}_{x^{\prime}}\left(\int_{0}^{1} U_{\infty}^{\prime} d y_{3}\right)=0 \quad \text { in } \omega, \\
\operatorname{div}_{z^{\prime}} U^{\prime}=0 \quad \text { in } \omega \times \mathbb{R}^{2} \times(0,1) .
\end{gathered}
$$

Proof. For the sake of completeness, we give a sketch of a proof, following, for instance, the proof of [11, Proposition 10.1]. 
By estimate (40), there exists $U \in L^{r}\left(\Omega ; L_{l o c}^{r}\left(\mathbb{R}^{2}\right)^{3}\right)$ such that, up to a subsequence, convergence (54) holds. Using the continuous embedding of $W^{1, r}\left(0,1 ; L^{r}(\omega)\right)$ into $L^{r}(\Omega)$, the definition of the local average (37) and according to [11, Proposition 1.25(ii)], we deduce from (45) the following convergence

$$
\sigma_{\varepsilon}^{-\frac{r}{r-1}} \bar{u}_{\varepsilon \delta} \rightarrow u \quad \text { weakly in } L^{r}(\Omega)^{3},
$$

so that

$$
\sigma_{\varepsilon}^{-\frac{r}{r-1}} \bar{u}_{\varepsilon \delta} \mathbf{1}_{\frac{1}{\delta} Y^{\prime}} \rightarrow u \quad \text { weakly in } L^{r}\left(\Omega ; L_{l o c}^{r}\left(\mathbb{R}^{2}\right)^{3}\right) .
$$

From the first estimate in (42) and estimate (43), there exists $W \in L^{r}\left(\Omega ; L^{r^{*}}\left(\mathbb{R}^{2}\right)^{3}\right)$ with $D_{z^{\prime}} W$ in $L^{r}\left(\omega \times \mathbb{R}^{2} \times\right.$ $(0,1))^{3 \times 2}$ such that

$$
\sigma_{\varepsilon}^{-\frac{r}{r-1}}\left(\hat{u}_{\varepsilon \delta}-\bar{u}_{\varepsilon \delta}\right) \mathbf{1}_{\frac{1}{\delta} Y^{\prime}} \rightarrow W \quad \text { weakly in } L^{r}\left(\Omega ; L^{r^{*}}\left(\mathbb{R}^{2}\right)^{3}\right),
$$

and

$$
\sigma_{\varepsilon}^{-\frac{r}{r-1}} D_{z^{\prime}} \hat{u}_{\varepsilon \delta} \mathbf{1}_{\frac{1}{\delta} Y^{\prime}} \rightarrow D_{z^{\prime}} W \quad \text { weakly in } L^{r}\left(\omega \times \mathbb{R}^{2} \times(0,1)\right)^{3 \times 2} .
$$

From (54), (58) and (59), one concludes

$$
U=W+u \quad \text { and } \quad D_{z^{\prime}} U=D_{z^{\prime}} W,
$$

and so, from convergence (60), we have that (55) holds. Since $\tilde{u}_{\varepsilon \delta}=0$ in $T_{\varepsilon \delta}$, we have $\hat{u}_{\varepsilon \delta}=0$ in $\omega \times Y_{s}$ and then, using convergence (54), we deduce

$$
U=0 \quad \text { in } \omega \times Y_{s} .
$$

Due to (59)-(62) and [11, Proposition 9.2], we have that $U \in \mathbf{L}_{Y_{s}^{\prime}}^{3}$ and $U_{\infty}=u$.

Since $U_{\infty}=u$, then (56) holds from the divergence condition (46). Next, from divergence condition $\operatorname{div}_{\sigma_{\varepsilon}} \tilde{u}_{\varepsilon \delta}=$ 0 in $\widetilde{\Omega}_{\varepsilon \delta}$ and the change of variables (31) and (38), we deduce

$$
(\varepsilon \delta)^{-1} \operatorname{div}_{z^{\prime}} \hat{u}_{\varepsilon \delta}^{\prime} \mathbf{1}_{\frac{1}{\delta} Y^{\prime}}+\sigma_{\varepsilon}^{-1} \partial_{y_{3}} \hat{u}_{\varepsilon \delta, 3} \mathbf{1}_{\frac{1}{\delta} Y^{\prime}}=0 \quad \text { in } \omega \times \mathbb{R}^{2} \times(0,1) .
$$

Multiplying by $\sigma_{\varepsilon}^{-\frac{r}{r-1}}(\varepsilon \delta)$, we get

$$
\sigma_{\varepsilon}^{-\frac{r}{r-1}} \operatorname{div}_{z^{\prime}} \hat{u}_{\varepsilon \delta}^{\prime} \mathbf{1}_{\frac{1}{\delta} Y^{\prime}}+\delta^{\frac{2}{r}} \sigma_{\varepsilon}^{-\frac{r}{r-1}} \partial_{y_{3}} \hat{u}_{\varepsilon \delta, 3} \mathbf{1}_{\frac{1}{\delta} Y^{\prime}}=0 \quad \text { in } \omega \times \mathbb{R}^{2} \times(0,1) .
$$

From the second estimate in (42) and convergence (54), we deduce that $\delta^{\frac{2}{r}} \sigma_{\varepsilon}^{-\frac{r}{r-1}} \partial_{y_{3}} \hat{u}_{\varepsilon \delta, 3}$ tends to zero, and so passing to the limit in (64) we get (57).

Finally, we prove that $U_{3}$ is independent of $y_{3}$. To do this, consider $\varphi \in C_{c}^{1}(\Omega)$ as test function in (63), which gives

$$
\int_{\omega \times \frac{1}{\delta} Y^{\prime} \times(0,1)} \partial_{y_{3}} \hat{u}_{\varepsilon \delta, 3} \varphi\left(x^{\prime}, y_{3}\right) d x^{\prime} d z^{\prime} d y_{3}=0 .
$$

Multiplying by $\sigma_{\varepsilon}^{-\frac{r}{r-1}}$, we have

$$
\int_{\omega \times \frac{1}{\delta} Y^{\prime} \times(0,1)} \sigma_{\varepsilon}^{-\frac{r}{r-1}} \hat{u}_{\varepsilon \delta, 3} \partial_{y_{3}} \varphi\left(x^{\prime}, y_{3}\right) d x^{\prime} d z^{\prime} d y_{3}=0,
$$

and from convergence (54), we get $U_{3}$ is independent of $y_{3}$. 


\section{Homogenized model: proof of the main Theorem}

In this section, we use previous compactness results to prove the main result of the paper. To do this, we need the following version of [11, Lemma 10.4] in order to choose an appropriate test function in the variational formulation of system (6) and then pass to the limit.

Lemma 6.1. Suppose $1 \leq r<+\infty$. Let $\varphi$ be in $\mathcal{D}\left(\Omega ; W_{\text {loc }}^{1, r}\left(\mathbb{R}^{2}\right)\right)$ such that $\nabla_{z^{\prime}} \varphi$ is in $\mathcal{D}\left(\Omega ; L^{r}\left(\mathbb{R}^{2}\right)^{2}\right)$ and has a compact support. We set

$$
\varphi_{\varepsilon \delta}\left(x^{\prime}, y_{3}\right)=\varphi\left(x^{\prime}, \frac{1}{\delta} \frac{x^{\prime}-\varepsilon \kappa\left(\frac{x^{\prime}}{\varepsilon}\right)}{\varepsilon}, y_{3}\right) \quad \text { in }\left(x^{\prime}, y_{3}\right) \in \Omega .
$$

By [11, Proposition 9.2], it has a limit at infinity denoted by $\varphi_{\infty} \in \mathcal{D}(\Omega)$. If $\delta$ is small enough, the function $\varphi_{\varepsilon \delta}$ belongs to $\mathcal{D}\left(0,1 ; W^{1, r}(\omega)\right)$ and

$$
\varphi_{\varepsilon \delta} \rightarrow \varphi_{\infty} \quad \text { strongly in } L^{r}(\Omega) .
$$

Remark 6.2 (Properties of test function). From the definition of $\varphi_{\varepsilon \delta}$ given in (65), we have

$$
\begin{aligned}
& \nabla_{x^{\prime}} \varphi_{\varepsilon \delta}\left(x^{\prime}, y_{3}\right)=\nabla_{x^{\prime}} \varphi\left(x^{\prime}, \frac{1}{\delta} \frac{x^{\prime}-\varepsilon \kappa\left(\frac{x^{\prime}}{\varepsilon}\right)}{\varepsilon}, y_{3}\right)+(\varepsilon \delta)^{-1} \nabla_{z^{\prime}} \varphi\left(x^{\prime}, \frac{1}{\delta} \frac{x^{\prime}-\varepsilon \kappa\left(\frac{x^{\prime}}{\varepsilon}\right)}{\varepsilon}, y_{3}\right), \\
& \partial_{y_{3}} \varphi_{\varepsilon \delta}\left(x^{\prime}, y_{3}\right)=\partial_{y_{3}} \varphi\left(x^{\prime}, \frac{1}{\delta} \frac{x^{\prime}-\varepsilon \kappa\left(\frac{x^{\prime}}{\varepsilon}\right)}{\varepsilon}, y_{3}\right) .
\end{aligned}
$$

Moreover, applying the unfolding operator (30), we have

$$
\hat{\varphi}_{\varepsilon \delta}\left(x^{\prime}, z^{\prime}, y_{3}\right)= \begin{cases}\varphi\left(x^{\prime}, z^{\prime}, y_{3}\right)+\Theta_{\varepsilon \delta}\left(x^{\prime}, z^{\prime}, y_{3}\right), & \text { if }\left(x^{\prime}, z^{\prime}, y_{3}\right) \in \omega \times \frac{1}{\delta} Y^{\prime} \times(0,1), \\ 0 & \text { otherwise, }\end{cases}
$$

with $\Theta_{\varepsilon \delta}\left(x^{\prime}, z^{\prime}, y_{3}\right)=\varphi\left(\varepsilon \kappa\left(\frac{x^{\prime}}{\varepsilon}\right)+\varepsilon \delta z^{\prime}, z^{\prime}, y_{3}\right)-\varphi\left(x^{\prime}, z^{\prime}, y_{3}\right)$. Consequently

$$
\nabla_{z^{\prime}} \hat{\varphi}_{\varepsilon \delta}\left(x^{\prime}, z^{\prime}, y_{3}\right)=\nabla_{z^{\prime}} \varphi\left(x^{\prime}, z^{\prime}, y_{3}\right)+\nabla_{z^{\prime}} \Theta_{\varepsilon \delta}\left(x^{\prime}, z^{\prime}, y_{3}\right) \quad \text { in } \omega \times \frac{1}{\delta} Y^{\prime} \times(0,1),
$$

where, from the mean value theorem applied to $\nabla_{z^{\prime}} \Theta_{\varepsilon \delta}$, the fact that $\left|\varepsilon \kappa\left(\frac{x^{\prime}}{\varepsilon}\right)+\varepsilon \delta z^{\prime}-x^{\prime}\right|<\varepsilon$ for $x^{\prime} \in Y_{k^{\prime}, \varepsilon}^{\prime}$, $k^{\prime} \in \mathcal{K}_{\varepsilon}$, and $\nabla_{z^{\prime}} \varphi \in \mathcal{D}\left(\Omega ; L^{r}\left(\mathbb{R}^{2}\right)^{2}\right)$, it holds

$$
\left\|\nabla_{z^{\prime}} \Theta_{\varepsilon \delta}\right\|_{L^{r}\left(\omega \times \mathbb{R}^{2} \times(0,1)\right)^{2}} \leq C \varepsilon .
$$

Proof of Theorem 2.1. The proof of the main result will be divided in two steps.

Step 1. Suppose $1<r<2$. We set

$$
\mathbb{W}=\left\{\begin{aligned}
\left(v^{\prime}, V^{\prime}\right) \in W^{1, r}\left(0,1 ; L^{r}(\omega)^{2}\right) \times \mathbf{L}_{Y_{s}^{\prime}}^{2} \quad: \quad V_{\infty}^{\prime}\left(x^{\prime}, y_{3}\right)=v^{\prime}\left(x^{\prime}, y_{3}\right) \quad \text { a.e. in }\left(x^{\prime}, y_{3}\right) \in \Omega, \\
\operatorname{div}_{z^{\prime}} V^{\prime}=0 \text { in } \omega \times \mathbb{R}^{2} \times(0,1), \quad \operatorname{div}_{x^{\prime}}\left(\int_{0}^{1} v^{\prime} d y_{3}\right)=0 \text { in } \omega, \quad\left(\int_{0}^{1} v^{\prime} d y_{3}\right) \cdot n=0 \text { on } \partial \omega,
\end{aligned}\right\} .
$$

To simplify the notation, we define the operator $S: \mathbb{R}_{\mathrm{sym}}^{3} \rightarrow \mathbb{R}_{\mathrm{sym}}^{3}$ by

$$
S(\xi)=|\xi|^{r-2} \xi, \quad \forall \xi \in \mathbb{R}_{\text {sym }}^{3 \times 3},
$$

and denote by $O_{\varepsilon}$ a generic real sequence, which tends to zero with $\varepsilon$ and $\delta$ and can change from line to line. 
In this step, we prove that the pair of functions $(u, U) \in \mathbb{W}$ given in Lemma 5.2 satisfies the homogenized problem

$$
\begin{aligned}
& \mu \int_{\Omega} S\left(\partial_{y_{3}}\left[u^{\prime}\right]\right): \partial_{y_{3}}\left[v^{\prime}\right] d x^{\prime} d y_{3}+\mu \int_{\omega \times\left(\mathbb{R}^{2} \backslash Y_{s}^{\prime}\right) \times(0,1)} S\left(\mathbb{D}_{z^{\prime}}\left[U^{\prime}\right]\right): \mathbb{D}_{z^{\prime}}\left[V^{\prime}\right] d x^{\prime} d z^{\prime} d y_{3} \\
& =\int_{\Omega} f^{\prime} \cdot v^{\prime} d x^{\prime} d y_{3}, \quad \forall\left(v^{\prime}, V^{\prime}\right) \in \mathbb{W} .
\end{aligned}
$$

To prove this, we consider $\varphi\left(x^{\prime}, z^{\prime}, y_{3}\right) \in \mathcal{D}\left(\Omega ; \mathbf{K}_{Y_{s}^{\prime}}^{3}\right)$ such that $D_{z^{\prime}} \varphi$ has a compact support, $\varphi_{3}$ is independent of $y_{3}$ and we define $\varphi_{\varepsilon \delta}$ by (65). From Lemma 6.1 , if $\delta$ is small enough the function $\varphi_{\varepsilon \delta}$ belongs to $\mathcal{D}\left(0,1 ; W^{1, r}(\omega)^{3}\right)$ and it has a limit at infinity $\varphi_{\infty} \in \mathcal{D}(\Omega)^{3}$ satisfying convergence (66).

Multiplying (6) by $\varphi_{\varepsilon \delta}$, taking into account the extensions of the velocity and pressure, integrating by parts and using (67), we have

$$
\begin{aligned}
& \mu \int_{\Omega} S\left(\mathbb{D}_{\sigma_{\varepsilon}}\left[\tilde{u}_{\varepsilon \delta}\right]\right):\left(\mathbb{D}_{x^{\prime}}[\varphi]+\sigma_{\varepsilon}^{-1} \partial_{y_{3}}[\varphi]\right) d x^{\prime} d y_{3}+\mu(\varepsilon \delta)^{-1} \int_{\Omega} S\left(\mathbb{D}_{\sigma_{\varepsilon}}\left[\tilde{u}_{\varepsilon \delta}\right]\right): \mathbb{D}_{z^{\prime}}[\varphi] d x^{\prime} d y_{3} \\
& -\int_{\Omega} \tilde{p}_{\varepsilon \delta} \operatorname{div}_{x^{\prime}} \varphi^{\prime} d x^{\prime} d y_{3}-(\varepsilon \delta)^{-1} \int_{\Omega} \tilde{p}_{\varepsilon \delta} \operatorname{div}_{z^{\prime}} \varphi^{\prime} d x^{\prime} d y_{3}=\int_{\widetilde{\Omega}_{\varepsilon \delta}} f^{\prime} \cdot \varphi^{\prime} d x^{\prime} d y_{3} .
\end{aligned}
$$

To simplify, from now on we use the following notation:

- $\varphi=\varphi\left(x^{\prime}, \frac{1}{\delta} \frac{x^{\prime}-\varepsilon \kappa\left(\frac{x^{\prime}}{\varepsilon}\right)}{\varepsilon}, y_{3}\right)$ in the integrals in $\Omega$,

- $\varphi=\varphi\left(x^{\prime}, z^{\prime}, y_{3}\right)$ in the integrals in $\omega \times \frac{1}{\delta} Y^{\prime} \times(0,1)$ obtained after applying the changes of variables $(31)$ and (38).

First, we analyze the first term in (71). Thus,

$$
\begin{aligned}
& \mu \int_{\Omega} S\left(\mathbb{D}_{x^{\prime}}\left[\tilde{u}_{\varepsilon \delta}\right]+\sigma_{\varepsilon}^{-1} \partial_{y_{3}}\left[\tilde{u}_{\varepsilon \delta}\right]\right):\left(\mathbb{D}_{x^{\prime}}[\varphi]+\sigma_{\varepsilon}^{-1} \partial_{y_{3}}[\varphi]\right) d x^{\prime} d y_{3} \\
& =\mu \int_{\Omega} S\left(\sigma_{\varepsilon} \mathbb{D}_{x^{\prime}}\left[\sigma_{\varepsilon}^{-\frac{r}{r-1}} \tilde{u}_{\varepsilon \delta}\right]+\partial_{y_{3}}\left[\sigma_{\varepsilon}^{-\frac{r}{r-1}} \tilde{u}_{\varepsilon \delta}\right]\right):\left(\sigma_{\varepsilon} \mathbb{D}_{x^{\prime}}[\varphi]+\partial_{y_{3}}[\varphi]\right) d x^{\prime} d y_{3} .
\end{aligned}
$$

Next, we analyze the second term in (71). By the changes of variables (31) and (38) and taking into account (68), we deduce

$$
\begin{aligned}
& \mu(\varepsilon \delta)^{-1} \int_{\Omega} S\left(\mathbb{D}_{x^{\prime}}\left[\tilde{u}_{\varepsilon \delta}\right]+\sigma_{\varepsilon}^{-1} \partial_{y_{3}}\left[\tilde{u}_{\varepsilon \delta}\right]\right): \mathbb{D}_{z^{\prime}}[\varphi] d x^{\prime} d y_{3} \\
& =\mu(\varepsilon \delta)^{-1} \delta^{2} \int_{\omega \times \frac{1}{\delta} Y^{\prime} \times(0,1)} S\left((\varepsilon \delta)^{-1} \mathbb{D}_{z^{\prime}}\left[\hat{u}_{\varepsilon \delta}\right]+\sigma_{\varepsilon}^{-1} \partial_{y_{3}}\left[\hat{u}_{\varepsilon \delta}\right]\right):\left(\mathbb{D}_{z^{\prime}}[\varphi]+\mathbb{D}_{z^{\prime}}\left[\Theta_{\varepsilon \delta}\right]\right) d x^{\prime} d z^{\prime} d y_{3} \\
& =\mu \int_{\omega \times \frac{1}{\delta} Y^{\prime} \times(0,1)} S\left(\mathbb{D}_{z^{\prime}}\left[\sigma_{\varepsilon}^{-\frac{r}{r-1}} \hat{u}_{\varepsilon \delta}\right]+\delta^{\frac{2}{r}} \partial_{y_{3}}\left[\sigma_{\varepsilon}^{-\frac{r}{r-1}} \hat{u}_{\varepsilon \delta}\right]\right):\left(\mathbb{D}_{z^{\prime}}[\varphi]+\mathbb{D}_{z^{\prime}}\left[\Theta_{\varepsilon \delta}\right]\right) d x^{\prime} d z^{\prime} d y_{3} .
\end{aligned}
$$

By Hölder's inequality, estimates given in (41) and taking into account (69), we have

$$
\left|\int_{\omega \times \frac{1}{\delta} Y^{\prime} \times(0,1)} S\left(\mathbb{D}_{z^{\prime}}\left[\sigma_{\varepsilon}^{-\frac{r}{r-1}} \hat{u}_{\varepsilon \delta}\right]+\delta^{\frac{2}{r}} \partial_{y_{3}}\left[\sigma_{\varepsilon}^{-\frac{r}{r-1}} \hat{u}_{\varepsilon \delta}\right]\right): \mathbb{D}_{z^{\prime}}\left[\Theta_{\varepsilon \delta}\right] d x^{\prime} d z^{\prime} d y_{3}\right| \leq C \varepsilon
$$


and then, (73) reads

$$
\begin{aligned}
& \mu(\varepsilon \delta)^{-1} \int_{\Omega} S\left(\mathbb{D}_{x^{\prime}}\left[\tilde{u}_{\varepsilon \delta}\right]+\sigma_{\varepsilon}^{-1} \partial_{y_{3}}\left[\tilde{u}_{\varepsilon \delta}\right]\right): \mathbb{D}_{z^{\prime}}[\varphi] d x^{\prime} d y_{3} \\
& =\mu \int_{\omega \times \frac{1}{\delta} Y^{\prime} \times(0,1)} S\left(\mathbb{D}_{z^{\prime}}\left[\sigma_{\varepsilon}^{-\frac{r}{r-1}} \hat{u}_{\varepsilon \delta}\right]+\delta^{\frac{2}{r}} \partial_{y_{3}}\left[\sigma_{\varepsilon}^{-\frac{r}{r-1}} \hat{u}_{\varepsilon \delta}\right]\right): \mathbb{D}_{z^{\prime}}[\varphi] d x^{\prime} d z^{\prime} d y_{3}+O_{\varepsilon} \\
& =\mu \int_{\omega \times \frac{1}{\delta} Y^{\prime} \times(0,1)} S\left(\mathbb{D}_{z^{\prime}}\left[\sigma_{\varepsilon}^{-\frac{r}{r-1}} \hat{u}_{\varepsilon \delta}\right]+\delta^{\frac{2}{r}} \partial_{y_{3}}\left[\sigma_{\varepsilon}^{-\frac{r}{r-1}} \hat{u}_{\varepsilon \delta}\right]\right):\left(\mathbb{D}_{z^{\prime}}[\varphi]+\delta^{\frac{2}{r}} \partial_{y_{3}}[\varphi]\right) d x^{\prime} d z^{\prime} d y_{3} \\
& -\mu \delta^{\frac{2}{r}} \int_{\omega \times \frac{1}{\delta} Y^{\prime} \times(0,1)} S\left(\mathbb{D}_{z^{\prime}}\left[\sigma_{\varepsilon}^{-\frac{r}{r-1}} \hat{u}_{\varepsilon \delta}\right]+\delta^{\frac{2}{r}} \partial_{y_{3}}\left[\sigma_{\varepsilon}^{-\frac{r}{r-1}} \hat{u}_{\varepsilon \delta}\right]\right): \partial_{y_{3}}[\varphi] d x^{\prime} d z^{\prime} d y_{3}+O_{\varepsilon} .
\end{aligned}
$$

Since $\varphi$ belongs to $\mathcal{D}\left(\Omega ; W_{l o c}^{1, r}\left(\mathbb{R}^{2}\right)^{3}\right)$, by Hölder's inequality and estimates in (41), we have

$$
\left|\delta^{\frac{2}{r}} \int_{\omega \times \frac{1}{\delta} Y^{\prime} \times(0,1)} S\left(\mathbb{D}_{z^{\prime}}\left[\sigma_{\varepsilon}^{-\frac{r}{r-1}} \hat{u}_{\varepsilon \delta}\right]+\delta^{\frac{2}{r}} \partial_{y_{3}}\left[\sigma_{\varepsilon}^{-\frac{r}{r-1}} \hat{u}_{\varepsilon \delta}\right]\right): \partial_{y_{3}}[\varphi] d x^{\prime} d z^{\prime} d y_{3}\right| \leq C \delta^{\frac{2}{r}}
$$

Then, (74) reads

$$
\begin{aligned}
& \mu(\varepsilon \delta)^{-1} \int_{\Omega} S\left(\mathbb{D}_{x^{\prime}}\left[\tilde{u}_{\varepsilon \delta}\right]+\sigma_{\varepsilon}^{-1} \partial_{y_{3}}\left[\tilde{u}_{\varepsilon \delta}\right]\right): \mathbb{D}_{z^{\prime}}[\varphi] d x^{\prime} d y_{3} \\
& =\mu \int_{\omega \times \frac{1}{\delta} Y^{\prime} \times(0,1)} S\left(\mathbb{D}_{z^{\prime}}\left[\sigma_{\varepsilon}^{-\frac{r}{r-1}} \hat{u}_{\varepsilon \delta}\right]+\delta^{\frac{2}{r}} \partial_{y_{3}}\left[\sigma_{\varepsilon}^{-\frac{r}{r-1}} \hat{u}_{\varepsilon \delta}\right]\right):\left(\mathbb{D}_{z^{\prime}}[\varphi]+\delta^{\frac{2}{r}} \partial_{y_{3}}[\varphi]\right) d x^{\prime} d z^{\prime} d y_{3}+O_{\varepsilon} .
\end{aligned}
$$

Now, we analyze the terms which involve the pressure. By the changes of variables (31) and (38) in the third and fourth terms in (71) and taking into account (68), we deduce

$$
\begin{aligned}
& -\int_{\Omega} \tilde{p}_{\varepsilon \delta} \operatorname{div}_{x^{\prime}} \varphi^{\prime} d x^{\prime} d y_{3}-(\varepsilon \delta)^{-1} \int_{\Omega} \tilde{p}_{\varepsilon \delta} \operatorname{div}_{z^{\prime}} \varphi^{\prime} d x^{\prime} d y_{3} \\
& =-\int_{\Omega} \tilde{p}_{\varepsilon \delta} \operatorname{div}_{x^{\prime}} \varphi^{\prime} d x^{\prime} d y_{3}-\delta^{2}(\varepsilon \delta)^{-1} \int_{\omega \times \mathbb{R}^{2} \times(0,1)} \hat{p}_{\varepsilon \delta}\left(\operatorname{div}_{z^{\prime}} \varphi^{\prime}+\operatorname{div}_{z^{\prime}} \Theta_{\varepsilon \delta}^{\prime}\right) d x^{\prime} d z^{\prime} d y_{3} .
\end{aligned}
$$

By Hölder's inequality, estimate (44) and taking into account (69), we have

$$
\left|\delta^{2}(\varepsilon \delta)^{-1} \int_{\omega \times \mathbb{R}^{2} \times(0,1)} \hat{p}_{\varepsilon \delta} \operatorname{div}_{z^{\prime}} \Theta_{\varepsilon \delta}^{\prime} d x^{\prime} d z^{\prime} d y_{3}\right| \leq C \delta^{\frac{2-r}{r}},
$$

and then, we have that $(76)$ reads

$$
\begin{aligned}
& -\int_{\Omega} \tilde{p}_{\varepsilon \delta} \operatorname{div}_{x^{\prime}} \varphi^{\prime} d x^{\prime} d y_{3}-(\varepsilon \delta)^{-1} \int_{\Omega} \tilde{p}_{\varepsilon \delta} \operatorname{div}_{z^{\prime}} \varphi^{\prime} d x^{\prime} d y_{3} \\
& =-\int_{\Omega} \tilde{p}_{\varepsilon \delta} \operatorname{div}_{x^{\prime}} \varphi^{\prime} d x^{\prime} d y_{3}-\delta^{2}(\varepsilon \delta)^{-1} \int_{\omega \times \mathbb{R}^{2} \times(0,1)} \hat{p}_{\varepsilon \delta} \operatorname{div}_{z^{\prime}} \varphi^{\prime} d x^{\prime} d z^{\prime} d y_{3}+O_{\varepsilon} .
\end{aligned}
$$

Then, taking into account (72), (75) and (77) in (71), we obtain

$$
\begin{aligned}
& \mu \int_{\Omega} S\left(\sigma_{\varepsilon} \mathbb{D}_{x^{\prime}}\left[\sigma_{\varepsilon}^{-\frac{r}{r-1}} \tilde{u}_{\varepsilon \delta}\right]+\partial_{y_{3}}\left[\sigma_{\varepsilon}^{-\frac{r}{r-1}} \tilde{u}_{\varepsilon \delta}\right]\right):\left(\sigma_{\varepsilon} \mathbb{D}_{x^{\prime}}[\varphi]+\partial_{y_{3}}[\varphi]\right) d x^{\prime} d y_{3} \\
& +\mu \int_{\omega \times \frac{1}{\delta} Y^{\prime} \times(0,1)} S\left(\mathbb{D}_{z^{\prime}}\left[\sigma_{\varepsilon}^{-\frac{r}{r-1}} \hat{u}_{\varepsilon \delta}\right]+\delta^{\frac{2}{r}} \partial_{y_{3}}\left[\sigma_{\varepsilon}^{-\frac{r}{r-1}} \hat{u}_{\varepsilon \delta}\right]\right):\left(\mathbb{D}_{z^{\prime}}[\varphi]+\delta^{\frac{2}{r}} \partial_{y_{3}}[\varphi]\right) d x^{\prime} d z^{\prime} d y_{3} \\
& -\int_{\Omega} \tilde{p}_{\varepsilon \delta} \operatorname{div}_{x^{\prime}} \varphi^{\prime} d x^{\prime} d y_{3}-\delta^{2}(\varepsilon \delta)^{-1} \int_{\omega \times \mathbb{R}^{2} \times(0,1)} \hat{p}_{\varepsilon \delta} \operatorname{div}_{z^{\prime}} \varphi^{\prime} d x^{\prime} d z^{\prime} d y_{3} \\
& =\int_{\widetilde{\Omega}_{\varepsilon \delta}} f^{\prime} \cdot \varphi^{\prime} d x^{\prime} d y_{3}+O_{\varepsilon} .
\end{aligned}
$$


Now, we consider $v=\left(v^{\prime}, 0\right) \in \mathcal{D}(\Omega)^{3}$ which satisfies the divergence condition $\operatorname{div}_{x^{\prime}} \int_{0}^{1} v^{\prime} d y_{3}=0$ in $\omega$. Also, we consider $V \in \mathcal{D}\left(\Omega ; \mathbf{K}_{Y_{s}^{\prime}}^{3}\right)$ such that $D_{z^{\prime}} V$ has a compact support, $V_{3}$ is independent of $y_{3}$ and $\operatorname{div}_{z^{\prime}} V^{\prime}=0$ in $\omega \times \mathbb{R}^{2} \times(0,1)$. We define $V_{\varepsilon \delta}$ by $(65)$, which has a limit at infinity denoted by $V_{\infty} \in \mathcal{D}(\Omega)^{3}$, and suppose $V_{\infty}\left(x^{\prime}, y_{3}\right)=v\left(x^{\prime}, y_{3}\right)$ a.e. in $\Omega$. Then, we choose as test function $\varphi$ in (78) the following functions

- $\varphi_{\varepsilon \delta}=V\left(x^{\prime}, \frac{1}{\delta} \frac{x^{\prime}-\varepsilon \kappa\left(\frac{x^{\prime}}{\varepsilon}\right)}{\varepsilon}, y_{3}\right)-\sigma_{\varepsilon}^{-\frac{r}{r-1}} \tilde{u}_{\varepsilon \delta}$ in the integrals in $\Omega$ and $\widetilde{\Omega}_{\varepsilon \delta}$,

- $\varphi_{\varepsilon \delta}=V\left(x^{\prime}, z^{\prime}, y_{3}\right)-\sigma_{\varepsilon}^{-\frac{r}{r-1}} \hat{u}_{\varepsilon \delta}$ in the integrals in $\omega \times \frac{1}{\delta} Y^{\prime} \times(0,1)$ and $\omega \times \mathbb{R}^{2} \times(0,1)$.

Taking into account (63), applying Hölder's inequality and using the second estimate in (42) and estimate (44), the fourth term in (78) satisfies

$$
\begin{aligned}
& \left|\delta^{2}(\varepsilon \delta)^{-1} \int_{\omega \times \mathbb{R}^{2} \times(0,1)} \hat{p}_{\varepsilon \delta} \operatorname{div}_{z^{\prime}} \varphi_{\varepsilon \delta}^{\prime} d x^{\prime} d z^{\prime} d y_{3}\right|=\left|\delta^{2}(\varepsilon \delta)^{-1} \int_{\omega \times \mathbb{R}^{2} \times(0,1)} \hat{p}_{\varepsilon \delta} \operatorname{div}_{z^{\prime}} \hat{u}_{\varepsilon \delta}^{\prime} d x^{\prime} d z^{\prime} d y_{3}\right| \\
& =\left|\delta^{2} \sigma_{\varepsilon}^{-1} \int_{\omega \times \mathbb{R}^{2} \times(0,1)} \hat{p}_{\varepsilon \delta} \partial_{y_{3}} \hat{u}_{\varepsilon \delta, 3} d x^{\prime} d z^{\prime} d y_{3}\right| \leq C \sigma_{\varepsilon}^{\frac{1}{r-1}},
\end{aligned}
$$

and so (78) reads

$$
\begin{aligned}
& \mu \int_{\Omega} S\left(\sigma_{\varepsilon} \mathbb{D}_{x^{\prime}}\left[\sigma_{\varepsilon}^{-\frac{r}{r-1}} \tilde{u}_{\varepsilon \delta}\right]+\partial_{y_{3}}\left[\sigma_{\varepsilon}^{-\frac{r}{r-1}} \tilde{u}_{\varepsilon \delta}\right]\right):\left(\sigma_{\varepsilon} \mathbb{D}_{x^{\prime}}\left[\varphi_{\varepsilon \delta}\right]+\partial_{y_{3}}\left[\varphi_{\varepsilon \delta}\right]\right) d x^{\prime} d y_{3} \\
& +\mu \int_{\omega \times \frac{1}{\delta} Y^{\prime} \times(0,1)} S\left(\mathbb{D}_{z^{\prime}}\left[\sigma_{\varepsilon}^{-\frac{r}{r-1}} \hat{u}_{\varepsilon \delta}\right]+\delta^{\frac{2}{r}} \partial_{y_{3}}\left[\sigma_{\varepsilon}^{-\frac{r}{r-1}} \hat{u}_{\varepsilon \delta}\right]\right):\left(\mathbb{D}_{z^{\prime}}\left[\varphi_{\varepsilon \delta}\right]+\delta^{\frac{2}{r}} \partial_{y_{3}}\left[\varphi_{\varepsilon \delta}\right]\right) d x^{\prime} d z^{\prime} d y_{3} \\
& -\int_{\Omega} \tilde{p}_{\varepsilon \delta} \operatorname{div}_{x^{\prime}} \varphi_{\varepsilon \delta}^{\prime} d x^{\prime} d y_{3}=\int_{\widetilde{\Omega}_{\varepsilon \delta}} f^{\prime} \cdot \varphi_{\varepsilon \delta}^{\prime} d x^{\prime} d y_{3}+O_{\varepsilon} .
\end{aligned}
$$

From this, we deduce

$$
\begin{aligned}
& -\mu \int_{\Omega}\left(S\left(\sigma_{\varepsilon} \mathbb{D}_{x^{\prime}}\left[\sigma_{\varepsilon}^{-\frac{r}{r-1}} \tilde{u}_{\varepsilon \delta}\right]+\partial_{y_{3}}\left[\sigma_{\varepsilon}^{-\frac{r}{r-1}} \tilde{u}_{\varepsilon \delta}\right]\right)-S\left(\sigma_{\varepsilon} \mathbb{D}_{x^{\prime}}[V]+\partial_{y_{3}}[V]\right)\right):\left(\sigma_{\varepsilon} \mathbb{D}_{x^{\prime}}\left[\varphi_{\varepsilon \delta}\right]+\partial_{y_{3}}\left[\varphi_{\varepsilon \delta}\right]\right) d x^{\prime} d y_{3} \\
& -\mu \int_{\Omega} S\left(\sigma_{\varepsilon} \mathbb{D}_{x^{\prime}}[V]+\partial_{y_{3}}[V]\right):\left(\sigma_{\varepsilon} \mathbb{D}_{x^{\prime}}\left[\varphi_{\varepsilon \delta}\right]+\partial_{y_{3}}\left[\varphi_{\varepsilon \delta}\right]\right) d x^{\prime} d y_{3} \\
& -\mu \int_{\omega \times \frac{1}{\delta} Y^{\prime} \times(0,1)}\left(S\left(\mathbb{D}_{z^{\prime}}\left[\sigma_{\varepsilon}^{-\frac{r}{r-1}} \hat{u}_{\varepsilon \delta}\right]+\delta^{\frac{2}{r}} \partial_{y_{3}}\left[\sigma_{\varepsilon}^{-\frac{r}{r-1}} \hat{u}_{\varepsilon \delta}\right]\right)-S\left(\mathbb{D}_{z^{\prime}}[V]+\delta^{\frac{2}{r}} \partial_{y_{3}}[V]\right)\right) \\
& :\left(\mathbb{D}_{z^{\prime}}\left[\varphi_{\varepsilon \delta}\right]+\delta^{\frac{2}{r}} \partial_{y_{3}}\left[\varphi_{\varepsilon \delta}\right]\right) d x^{\prime} d z^{\prime} d y_{3} \\
& -\mu \int_{\omega \times \frac{1}{\delta} Y^{\prime} \times(0,1)} S\left(\mathbb{D}_{z^{\prime}}[V]+\delta^{\frac{2}{r}} \partial_{y_{3}}[V]\right):\left(\mathbb{D}_{z^{\prime}}\left[\varphi_{\varepsilon \delta}\right]+\delta^{\frac{2}{r}} \partial_{y_{3}}\left[\varphi_{\varepsilon \delta}\right]\right) d x^{\prime} d z^{\prime} d y_{3} \\
& +\int_{\Omega} \tilde{p}_{\varepsilon \delta} \operatorname{div}_{x^{\prime}} \varphi_{\varepsilon \delta}^{\prime} d x^{\prime} d y_{3}=-\int_{\widetilde{\Omega}_{\varepsilon \delta}} f^{\prime} \cdot \varphi_{\varepsilon \delta}^{\prime} d x^{\prime} d y_{3}+O_{\varepsilon} .
\end{aligned}
$$

Since the operator $S$ is monotone, i.e. $(S(\xi)-S(\zeta)):(\xi-\zeta) \geq 0$ for every $\xi, \zeta \in \mathbb{R}^{3}$, we have

$$
\begin{aligned}
& \mu \int_{\Omega} S\left(\sigma_{\varepsilon} \mathbb{D}_{x^{\prime}}[V]+\partial_{y_{3}}[V]\right):\left(\sigma_{\varepsilon} \mathbb{D}_{x^{\prime}}\left[\varphi_{\varepsilon \delta}\right]+\partial_{y_{3}}\left[\varphi_{\varepsilon \delta}\right]\right) d x^{\prime} d y_{3} \\
& +\mu \int_{\omega \times \frac{1}{\delta} Y^{\prime} \times(0,1)} S\left(\mathbb{D}_{z^{\prime}}[V]+\delta^{\frac{2}{r}} \partial_{y_{3}}[V]\right):\left(\mathbb{D}_{z^{\prime}}\left[\varphi_{\varepsilon \delta}\right]+\delta^{\frac{2}{r}} \partial_{y_{3}}\left[\varphi_{\varepsilon \delta}\right]\right) d x^{\prime} d z^{\prime} d y_{3} \\
& -\int_{\Omega} \tilde{p}_{\varepsilon \delta} \operatorname{div}_{x^{\prime}} \varphi_{\varepsilon \delta}^{\prime} d x^{\prime} d y_{3} \geq \int_{\widetilde{\Omega}_{\varepsilon \delta}} f^{\prime} \cdot \varphi_{\varepsilon \delta}^{\prime} d x^{\prime} d y_{3}+O_{\varepsilon} .
\end{aligned}
$$


Then, passing to the limit by taking into account that $\sigma_{\varepsilon}$ and $\delta^{\frac{2}{r}}$ tend to 0 and using convergences (45), (47), (55) and (66), we obtain

$$
\begin{aligned}
& \mu \int_{\Omega} S\left(\partial_{y_{3}}\left[V_{\infty}^{\prime}\right]\right):\left(\partial_{y_{3}}\left[V_{\infty}^{\prime}-u^{\prime}\right]\right) d x^{\prime} d y_{3}+\mu \int_{\omega \times\left(\mathbb{R}^{2} \backslash Y_{s}^{\prime}\right) \times(0,1)} S\left(\mathbb{D}_{z^{\prime}}[V]\right): \mathbb{D}_{z^{\prime}}[V-U] d x^{\prime} d z^{\prime} d y_{3} \\
& -\int_{\Omega} p \operatorname{div}_{x^{\prime}}\left(V_{\infty}^{\prime}-u^{\prime}\right) d x^{\prime} d y_{3} \geq \int_{\Omega} f^{\prime} \cdot\left(V_{\infty}^{\prime}-u^{\prime}\right) d x^{\prime} d y_{3} .
\end{aligned}
$$

Since $p$ does not depend on $y_{3}$, according to $V_{\infty}^{\prime}=v^{\prime}$ in $\Omega$ and $\operatorname{div}_{x^{\prime}} \int_{0}^{1} v^{\prime} d y_{3}=0$ in $\omega$, we have

$$
\int_{\Omega} p \operatorname{div}_{x^{\prime}}\left(V_{\infty}^{\prime}-u^{\prime}\right) d x^{\prime} d y_{3}=\int_{\Omega} p \operatorname{div}_{x^{\prime}}\left(v^{\prime}-u^{\prime}\right) d x^{\prime} d y_{3}=\int_{\omega} p \operatorname{div}_{x^{\prime}}\left(\int_{0}^{1}\left(v^{\prime}-u^{\prime}\right) d y_{3}\right) d x^{\prime}=0,
$$

so we get

$$
\begin{aligned}
& \mu \int_{\Omega} S\left(\partial_{y_{3}}\left[V_{\infty}^{\prime}\right]\right):\left(\partial_{y_{3}}\left[V_{\infty}^{\prime}-u^{\prime}\right]\right) d x^{\prime} d y_{3}+\mu \int_{\omega \times\left(\mathbb{R}^{2} \backslash Y_{s}^{\prime}\right) \times(0,1)} S\left(\mathbb{D}_{z^{\prime}}[V]\right): \mathbb{D}_{z^{\prime}}[V-U] d x^{\prime} d z^{\prime} d y_{3} \\
& \geq \int_{\Omega} f^{\prime} \cdot\left(V_{\infty}^{\prime}-u^{\prime}\right) d x^{\prime} d y_{3},
\end{aligned}
$$

which, due to Minty Lemma [17, Chapter 3, Lemma 1.2], is equivalent to

$$
\mu \int_{\Omega} S\left(\partial_{y_{3}}\left[u^{\prime}\right]\right): \partial_{y_{3}}\left[V_{\infty}^{\prime}\right] d x^{\prime} d y_{3}+\mu \int_{\omega \times\left(\mathbb{R}^{2} \backslash Y_{s}^{\prime}\right) \times(0,1)} S\left(\mathbb{D}_{z^{\prime}}[U]\right): \mathbb{D}_{z^{\prime}}[V] d x^{\prime} d z^{\prime} d y_{3}=\int_{\Omega} f^{\prime} \cdot V_{\infty}^{\prime} d x^{\prime} d y_{3}
$$

Taking in the previous variational equation $W=\left(0,0, U_{3}\right)$ as test function, we have

$$
\int_{\mathbb{R}^{2} \backslash Y_{s}^{\prime}}\left|\mathbb{D}_{z^{\prime}}[U]\right|^{r-2} \mathbb{D}_{z^{\prime}}[U]: \mathbb{D}_{z^{\prime}}[W] d z^{\prime}=0, \quad \text { a.e. in } \Omega,
$$

and since $U_{3} \in \mathbf{L}_{Y_{s}^{\prime}}$, from [11, Proposition 9.2], we have

$$
\left\|U_{3}-U_{\infty, 3}\right\|_{L^{r^{*}\left(\mathbb{R}^{2}\right)}}^{r} \leq C\left\|\mathbb{D}_{z^{\prime}}\left[U_{3}\right]\right\|_{L^{r}\left(\mathbb{R}^{2}\right)^{2}}^{r} \text { a.e. in } \Omega .
$$

From Lemma 5.2, we know that $U_{\infty, 3}=0$ in $\Omega$ and so, we deduce

$$
\left\|U_{3}\right\|_{L^{r^{*}}\left(\mathbb{R}^{2}\right)}^{r} \leq C\left\|\mathbb{D}_{z^{\prime}}\left[U_{3}\right]\right\|_{L^{r}\left(\mathbb{R}^{2}\right)^{2}}^{r} \leq\left. C\left|\int_{\mathbb{R}^{2} \backslash Y_{s}^{\prime}}\right| \mathbb{D}_{z^{\prime}}[U]\right|^{r-2} \mathbb{D}_{z^{\prime}}[U]: \mathbb{D}_{z^{\prime}}[W] d z^{\prime} \mid=0,
$$

which implies $U_{3}=0$. Then, the variational equation (80) reads

$$
\mu \int_{\Omega} S\left(\partial_{y_{3}}\left[u^{\prime}\right]\right): \partial_{y_{3}}\left[v^{\prime}\right] d x^{\prime} d y_{3}+\mu \int_{\omega \times\left(\mathbb{R}^{2} \backslash Y_{s}^{\prime}\right) \times(0,1)} S\left(\mathbb{D}_{z^{\prime}}\left[U^{\prime}\right]\right): \mathbb{D}_{z^{\prime}}\left[V^{\prime}\right] d x^{\prime} d z^{\prime} d y_{3}=\int_{\Omega} f^{\prime} \cdot v^{\prime} d x^{\prime} d y_{3},
$$

which by density gives $(70)$ for every $\left(v^{\prime}, V^{\prime}\right) \in \mathbb{W}$.

Step 2. Let us obtain a problem for $u^{\prime}$ identifying $U^{\prime}$ in (70). For this purpose, for every $\xi^{\prime} \in \mathbb{R}^{2}$ we consider the unique solution of the auxiliary problem (9) denoted by $\left(w^{\xi^{\prime}}, \pi^{\xi^{\prime}}\right) \in D^{1, r}\left(\mathbb{R}^{2} \backslash Y_{s}^{\prime}\right)^{2} \times L^{r^{\prime}}\left(\mathbb{R}^{2} \backslash Y_{s}^{\prime}\right) / \mathbb{R}$ and the drag force function $\mathcal{G}\left(\xi^{\prime}\right)$ given by (8). We remark that then $w^{\xi^{\prime}} \in W_{l o c}^{1, r}\left(\mathbb{R}^{2} \backslash Y_{s}^{\prime}\right)^{2}$, see [16, Lemma II.6.1].

Thus, we can take in $(70)$ the pairs of functions $\left(u^{\prime}, U^{\prime}\right),\left(v^{\prime}, V^{\prime}\right) \in \mathbb{W}$ in the following way

$$
U^{\prime}\left(x^{\prime}, z^{\prime}, y_{3}\right)=w^{u^{\prime}\left(x^{\prime}, y_{3}\right)}\left(z^{\prime}\right), \quad V^{\prime}\left(x^{\prime}, z^{\prime}, y_{3}\right)=w^{v^{\prime}\left(x^{\prime}, y_{3}\right)}\left(z^{\prime}\right) \quad \text { a.e. } \omega \times\left(\mathbb{R}^{2} \backslash Y_{s}^{\prime}\right) \times(0,1),
$$


and then, we deduce

$$
\mu \int_{\Omega} S\left(\partial_{y_{3}}\left[u^{\prime}\right]\right): \partial_{y_{3}}\left[v^{\prime}\right] d x^{\prime} d y_{3}+\mu \int_{\Omega} \mathcal{G}\left(u^{\prime}\right) \cdot v^{\prime} d x^{\prime} d y_{3}=\int_{\Omega} f^{\prime} \cdot v^{\prime} d x^{\prime} d y_{3}, \quad \forall v^{\prime} \in \mathcal{V},
$$

where

$$
\mathcal{V}=\left\{v^{\prime} \in W^{1, r}\left(0,1 ; L^{r}(\omega)^{2}\right): \operatorname{div}_{x^{\prime}}\left(\int_{0}^{1} v^{\prime}\left(x^{\prime}, y_{3}\right) d y_{3}\right)=0 \text { in } \omega,\left(\int_{0}^{1} v^{\prime}\left(x^{\prime}, y_{3}\right) d y_{3}\right) \cdot n=0 \text { on } \partial \omega\right\} .
$$

From the properties of $\mathcal{G}$ given in Remark 2.3 and following [15, Lemma 4.4], the variational formulation (81) has a unique solution $u^{\prime} \in \mathcal{V}$. Finally, taking into account that

$$
\left|\partial_{y_{3}}\left[u^{\prime}\right]\right|^{r-2}=\left|\operatorname{Tr}\left(\partial_{y_{3}}\left[u^{\prime}\right] \partial_{y_{3}}^{t}\left[u^{\prime}\right]\right)\right|^{\frac{r}{2}-1},
$$

which implies

$$
S\left(\partial_{y_{3}}\left[u^{\prime}\right]\right)=2^{-\frac{r}{2}} S\left(\partial_{y_{3}} u^{\prime}\right),
$$

we deduce that there exists $q \in L^{r^{\prime}}(\omega) / \mathbb{R}$ such that the variational formulation (81) is equivalent to system (7). It remains to prove that such function $q$ coincides with the pressure $p$ given in Lemma 5.1. This can be easily done by proceeding as in Step 1 but considering test functions without satisfying the divergence condition $\operatorname{div}_{x^{\prime}}$, and identifying limits. Since problem $(7)$ has a unique solution, then the entire sequence $\left(\tilde{u}_{\varepsilon \delta}, \tilde{p}_{\varepsilon \delta}\right)$ converges to $(u, p)$. This finishes the proof.

\section{References}

[1] G. Allaire, Homogenization of the Navier-Stokes equations in open sets perforated with tiny holes I. Abstract framework, a volume distribution of holes, Arch. Ration. Mech. Anal., 113, (1991), 209 - 259.

[2] G. Allaire, One-phase Newtonian flow, in: Homogenization and Porous Media, Interdisciplinary Applied Mathematics Series, 6, Springer-Verlag, New York, (1997), 77-94.

[3] M. Anguiano, Homogenization of a non-stationary non-Newtonian flow in a porous medium containing a thin fissure, Eur. J. Appl. Math., 30(2), (2019), 248-277.

[4] M. Anguiano and F.J. Suárez-Grau, Homogenization of an incompressible non-newtonian flow through a thin porous medium, Z. Angew. Math. Phys., (2017), 68:45.

[5] M. Anguiano and F.J. Suárez-Grau, The transition between the Navier-Stokes equations to the Darcy equation in a thin porous medium, Mediterr. J. Math., (2018), 15:45.

[6] M. Anguiano and F.J. Suárez-Grau, Nonlinear Reynolds equations for non-Newtonian thin-film fluid flows over a rough boundary, IMA J. Appl. Math., 84, (2019), 63-95.

[7] M. Boukrouche and R. El Mir, Asymptotic analysis of a non-Newtonian fluid in a thin domain with Tresca law. Nonlinear Anal., 59, (2004), 85-105.

[8] A. Bourgeat and A. Mikelić, Homogenization of a polymer flow through a porous medium, Nonlinear Anal., 26, (1996), 1221-1253.

[9] A. Bourgeat, O. Gipouloux and A. Mikelić, Filtration law for polymer flow through porous media, Multiscale Model. Sim., 1, (2003), 432-457.

[10] A. Brillard, Asymptotic analysis of incompressible and viscous fluid flow through porous media. Brinkman's law via epi-convergence methods, in: Ann. Fac. Sci. Toulouse, Math. Toulouse: Université Paul Sabatier, Faculté des Sciences, 8, (1986-1987), 225-252. 
[11] D. Cioranescu, A. Damlamian and G. Griso, The periodic unfolding method: theory and applications to partial differential problems, Series in Contemporary Mathematics, 3, Springer, Singapore, 2018.

[12] A. Duvnjak, Derivation of Non-linear Reynolds-type Problem for Lubrication of a Rotating Shaft, ZAMM-Z. Angew. Math. Mech., 82, (2002), 317-333.

[13] J. Fabricius, J.G. I. Hellström, T.S. Lundström, E. Miroshnikova and P. Wall, Darcy's law for flow in a periodic thin porous medium confined between two parallel plates, Transp. Porous Med., 115, (2016), $473-493$.

[14] T. Fratrović and E. Marušić-Paloka, Low-volume-fraction limit for polymer fluids, J. Math. Anal. Appl. 373 (2011) 399-409.

[15] T. Fratrović and E. Marušić-Paloka, Nonlinear Brinkman-type law as a critical case in the polymer fluid filtration, Appl. Anal. 95 (2016) 562-583.

[16] G.P. Galdi, An introduction to the mathematical theory of the Navier-Stokes equations, Springer-Verlag, 1994.

[17] J.L. Lions, Quelques méthodes de résolution des problèmes aux limites non linéaires, Dunod, GauthierVillars, Paris, 1969.

[18] E. Marušić-Paloka, On the Stokes paradox for power-law fluids, ZAMM-Z. Angew. Math. Mech., 81, (2001), $31-36$.

[19] A. Mikelić and R. Tapiero, Mathematical derivation of power law describing polymer flow through a thin slab, RAIRO-Model Math. Anal. Numer., 29, (1995), 3-21.

[20] A. Mikelić, Non-Newtonian Flow, in: Homogenization and Porous Media, Interdisciplinary Applied Mathematics Series, 6, Springer-Verlag, New York, (1997), 45-68.

[21] A. Mikelić, An introduction to the homogenization modeling of non-Newtonian and electrokinetic flows in porous media, in: Non-Newtonian Fluid Mechanics and Complex Flows. Lecture Notes in Mathematics, 2212, Springer, 2018.

[22] F.J. Suárez-Grau, Asymptotic behavior of a non-Newtonian flow in a thin domain with Navier law on a rough boundary. Nonlinear Anal., 117, (2015), 99-123. 